\title{
Mixed contact problems in plane elasticity $\left.\mathbf{U}^{\mathbf{1}}\right)$
}

\section{J. MAUL}

Der Beitrag ist die direkte Fortführung des Artikels [2] des Verfassers. Zunä̀chst wird der Index des in [2] erhaltenen singulären Integralgleichungssystems mit unstetigen Koeffizienten nach der Theoric von N. P. Vekua berechnet. Die Rechnungen werden durch einige zuvor bewiesene Folgerungen aus den Ergebnissen von Vekua vereinfacht. Die Existenz von ${ }^{*}$-regulären Lösungen der Kontaktaufgabe wird bewiesen unter der Voraussetzung, daß die physikalischen Lösbarkeitsbedingungen erfüllt sind. Dié Existenz von $\varepsilon^{*}$ - bzw. $\varepsilon$-regulären Lösungen setzen das Erfülltsein züsätzlicher Lösbarkeitsbedingungén voraus, deren Anzahl vom Index des singulären Integralgleichungssystems abhängt.

Продолжается изучение проблемы из статьи [2] автора. Индекс полученно в [2] системы сингулярных интегральных уравнений с разрывным коеффицентани вычисляется по теории Н..II. Векуа. Вычисления облегчаютсн нскоторыми доказаниыми в работе следствиями резуліьтатов Векуа. Доказывается, что физические условия разрешимости являются необходимыми и достаточными для существования *-регулярного решения контактной задачи. Существование $\dot{\varepsilon}^{*}$-регулярного и $\varepsilon$-регуляриого репений обеспечивается дополнительными условиями, число которых зависит от инденса системы сингулярных интегральных уравненй.

The paper continues the considerations in the previous one [2] of the author. Using N.P. Vekua's theory, the index of the singular integral equation system with discontinuous coefficients obtained in [2] is calculated. By establishing some corollaries from the theory of Vekua, the calculations can be simplified. Furthermore, the existence of $\bar{a}^{*}$-regular solution of the contact problem is proved, provided that the necessary physical conditions are fulfilled. The existence of $\varepsilon^{*}$ - or $\varepsilon$-regular solutions requires supplementary conditions, whose number depends on the index of the singular integral equation system.

This paper is the direct continuation of the considerations in its first part [2]. In [2] a general mixed contact problem of plane elasticity has been rigorously stated (problems $C^{*}, C_{\varepsilon}$ and $C_{\varepsilon}{ }^{*}$ of [2]). Moreover, the problem of uniqueness has been investigated. Using before-defined regularity conceptions and some properties of the single layer potential, the contact problem is, by certain manipulations, reduced to the integral equation

$$
, \boldsymbol{\Omega}_{p} \mathcal{A} \Phi=\mathrm{w}+A_{1} \boldsymbol{\Omega}_{p} \mathbf{w}_{1}+A_{2} \boldsymbol{\Omega}_{p} \mathbf{w}_{z}, \quad, A_{i}-\text { arbitrary constants. }
$$

The aim of the present paper is the exact analysis of (5.10) including the calculation of the index. For this purpose, the necessary tools of integral equations theory by N. P. VEkUA [7] and MUSkmelishinili [6] with some additional remarks are placed at disposal in $\S 7$. The relatively extensive and irksome considerations in connection with the determination of the index are carried out in $\S \S 8-10$. $\S \S 11$ and 12 deal with the contact problem $C^{*}$ in the class of *-regular solutions; namely, $\S 11$ is concerned with the case of uniqueness, but $\S 12$ with selected cases having eigen-

.1) Teil I erschien in dieser Zeitschrift in $\mathrm{H} .3$ (1983). 
solutions: In the smaller classes of $\varepsilon$ - and $\varepsilon^{*}$-regular vectors (comp. with $\S 2$ ) the contact problem $\left(C_{\varepsilon}\right.$ and $\left.C_{e}{ }^{*}\right)$ is studied in $\S 13$.

The paper is furnished with its own bibliographic references. Formulas, theorems and lemmata of [2] are referred to as in the following patterns: $(5.10)=$ formula (5.10) of $\S 5$ in [2]; Theorem $4.1=$ Theorem 1 in $\S 4$ of [2] etc. Supplementary bibliographic informations can be found in [2].

\section{$\S 7$ Singular integral equation systems with discontinuous coefficients}

The theory of singular integral equation systems with discontinuous coefficients

$$
\mathbf{A}(t) \mathbf{\Phi}(t)+\frac{\mathbf{B}(t)}{\pi i} \int_{L} \frac{\dot{\Phi}(\tau)}{\tau-t} d \tau+\int_{L} \mathbf{K}(t, \tau) \boldsymbol{\Phi}(\tau) d \tau=\mathbf{F}(t), . . t \in L
$$

has been stated by N. P. VEKJA in [7]. For our purpose, (7.1) shall be studied under the following assumptions:

Let $L$ be a simple closed curve of the class $C^{1, \beta}(0<\beta \leqq 1)$ in the complex plane $\mathbf{C}$. Let $m$ pairwise disjoint points $a_{1}, \ldots, a_{m}$ (so-called "nodes") be arranged on $L$ in counter-clockwise sense. Let $\mathbf{A}, \mathbf{B}, \mathbf{K}$ be $(n, n)$-matrices $(n \geqq 1)$ and $\mathbf{\Phi}, \mathbf{F} n$-vectors. Let the components of $\mathbf{A}, \mathbf{B}$ belong to the class $H_{0}$ and, additionally, to the class. $C^{0,1}\left(a_{i}, a_{i+1}\right) \quad(i=1, \ldots, m)$. The components of the matrix $\mathbf{K}(t, \tau)$, are supposed to belong to $H_{\varepsilon}$ in both variables, but those of $\mathbf{F}(t)$, also to $H_{\varepsilon}$. The solution $\boldsymbol{\Phi}$ of (7.1) is sought in the class $H^{*}$ or in subclasses still to be defined.

Now a short summary without proofs of Vekua's results concerning the system (7.1) should be given:

(7.1) is called of regular type if

$$
\operatorname{det}(\mathbf{A}+\mathbf{B}) \neq \mathbf{0}, \quad \operatorname{det}(\mathbf{A}-\mathbf{B}) \neq 0 \quad \text { everywhere on } L .
$$

(In the nodes $a_{i}$ this relation is to be understood as applying to the one-sided limits.)

The adjoint system is

$$
\mathbf{A}^{\top}(t) \Psi(t)-\frac{1}{\pi i} \int_{L} \frac{\mathbf{B}^{\top}(\tau) \Psi(\tau)}{\tau-t} d \tau+\int_{L} \mathbf{K}^{\top}(\tau, t) \Psi(\tau) d \tau=\mathbf{G}(t),
$$

where the matricés $\mathbf{A}^{\top}, \mathbf{B}^{\top}$ and $\mathbf{K}^{\top}$ are obtained from $\mathbf{A}, \mathbf{B}$ and $\mathbf{K}$ by transposition.

Let $(7.1)$ be of regular type. Setting

$$
g(t)=[\mathbf{A}(t)+\mathbf{B}(t)]^{-1}[\mathbf{A}(t)-\mathbf{B}(t)],
$$

let us define the matrices

$$
\gamma^{i}=\gamma\left(a_{i}\right)=\mathrm{g}^{-1}\left(a_{i}+0\right) \mathrm{g}\left(a_{i}-0\right), \quad(i=1, \ldots, m)
$$

in each node $a_{i}$. Let us consider the characteristic equations

$$
\operatorname{det}\left[\gamma^{i}-\lambda \mathbf{E}\right]=0 \quad(j=1, \ldots, m)
$$

(E - $(n, n)$-unit matrix). Let $\lambda_{1}{ }^{j}, \ldots, \lambda_{n}{ }^{j}$ be the roots of (7.6), counted with their multiplicity. In virtue of

$$
\prod_{i=1}^{n} \lambda_{l}{ }^{j}=\operatorname{det} g^{-1}\left(a_{i}+0\right) \operatorname{det} \mathrm{g}\left(a_{i}-0\right) \neq 0
$$

we have $\lambda_{l}{ }^{j} \neq 0$ for every $l=1, \ldots, n, j=1, \ldots, m$. 
The complex numbers $\varrho_{l}^{j}$ are defined by

$$
\lambda_{l}{ }^{j}=\mathrm{e}^{2 \pi i e_{l}{ }^{\prime}} \quad \text { and } \quad-1<\operatorname{Re} \varrho_{l}{ }^{j}<1 \quad(l=1, \ldots, n) \ldots
$$

If $\operatorname{Re} \varrho_{l}^{j}=0$ for every $l=1, \ldots, n$, the node $a_{j}$ is called special, and otherwise, nonspecial.

Now, we agree to choose the numbers $\varrho_{l}^{j}$ for fixed $j$ in such a way that either Re $\varrho_{l}{ }^{j} \leqq 0$ for all $l=1, \ldots, n$ or $\operatorname{Re} \varrho_{i}{ }^{j} \geqq 0$ for all $l=1, \ldots, n$.

Let the nodes $a_{j_{1}}, \ldots, a_{j_{r}}$ be nonspecial. We divide the set $\left\{a_{j_{1}}, \ldots, a_{j_{r}}\right\}$ into two subsets: $a_{j_{1}}, \ldots, a_{j_{a}}$ and $a_{j_{1}}, \ldots a_{j_{q}+1}, \ldots, a_{j_{r}}$. Stipulating $\operatorname{Re} \varrho_{l} j \geqq 0$ for $j=j_{1}, \ldots, j_{q}$ and $\operatorname{Re} \varrho_{l}{ }^{j} \leqq 0$ for $j=j_{q+1}, \ldots, j_{r}(l=1, \ldots, n)$, respectively, we give the following definitions.

Definition 1: 1 . The vector $\boldsymbol{\Phi}$ belongs to the class $h=h\left(a_{j_{1}}, \ldots, a_{j_{e}}\right)$ if $\boldsymbol{\Phi} \in H^{*}$ and $\boldsymbol{\Phi} \in H_{\varepsilon}$ in the neighbourhood of the nodes $a_{j_{1}}, \ldots, a_{j_{a}}, a_{j_{r+1}}, \ldots, a_{j_{n}}$.

2. The class $h\left(a_{j_{r+1}}, \ldots, a_{j_{r}}\right)$ is called adjoint to $h\left(a_{j_{1}}, \ldots, a_{j_{q}}\right)$.

In short notation, we will speak of the class $h$, which denotes a certain class $h\left(a_{j_{1}}, \ldots\right.$, $\left.a_{j_{q}}\right)$. Furthermore, the class $h\left(a_{j_{1}}, \ldots, a_{j_{r}}\right)$ is called $h_{\varepsilon}$, and $h^{*}$, the related adjoint class. Obviously, the class $h^{*}$ is the largest, and $h_{\varepsilon}$, the smallest one. In the case where $r=m$ (i.e. special nodes do not exist) we have $h^{*}={ }_{1} H^{*}$ and $h_{\varepsilon}=H_{\epsilon}$.

In the following theorem the main result of $\mathrm{N}$. P. Vekua concerning equations of type (7.1) is formulated. The proof can be found in [7: pp. 95-160].

Theorem 1: Let $\mathbf{F}, \mathbf{G} \in H_{\varepsilon}$. Let the matrices $\mathbf{A}, \mathbf{B}$ and $\mathbf{K}$ satisfy the above-mentioned assumptions.. Then Noether's theorems are valid for the equations (7.1) and (7.3) with respect to solvability in the class $h\left(a_{j_{1}}, \ldots, a_{j_{q}}\right)$ with the related adjoint class $h\left(a_{j_{a+1}}, \ldots, a_{j_{r}}\right)$, respectively.

The index $x=\varkappa_{h\left(a_{j_{1}}, \ldots, a_{j_{a}}\right)}=\varkappa_{h}$ of (7.1) can be calculated by the formula

$$
\varkappa_{h}=\frac{1}{2 \pi}\left[\arg \frac{\operatorname{det} g(t)}{\prod_{j=1}^{m} \prod_{l=1}^{n}\left(t-z_{0}\right)^{e_{l}}}\right]_{L}
$$

Here $z_{0}$ is an arbitrary point located within the bounded domain $D$ with $\partial D=L$. The numbers $\varrho_{l}{ }^{j}$ are to be chosen in accordance with Definition 1, and the branches of the functions $\left(t-z_{0}\right)^{e_{i}}$ are to be defined in such a way that these functions are holomorphic with respect to $t$ in the complex plane $C$, which is cut out across the line $z_{0} a_{j} \infty$. The symbol $[\div]_{L}$ means the increment on $L$ of the function in square brackets.

For large $n$, the actual calculation of $\lambda_{l}{ }^{i}$ is, in general, very extensive and irksome. Therefore the following lemma will be very useful.

Lemma 1: Let the matrix $\mathbf{A}$ consist of real elements and the mairix $\mathbf{B}$ of purely imaginary ones. Let equation (7.1) be of regular type. Then the following relations hold:

a) $|\operatorname{det} g|=1, \quad \cdot\left|\operatorname{det} \gamma\left(a_{j}\right)\right|=1 \quad(j=1, \ldots, m)$.

b) $\mathbf{g}^{-1}=\overline{\mathbf{g}}, \quad \operatorname{det}\left(\gamma^{-1}\right)=\operatorname{det} \bar{\gamma}$.

c) If $f_{j}\left(\lambda_{0}\right)=\operatorname{det}\left[\gamma\left(a_{j}\right)-\lambda \mathbf{E}\right]=b_{0}{ }^{j} \lambda^{n}+b_{1}{ }^{j \lambda^{n-1}}+\cdots+b_{n}{ }^{j}$. is the characteristic equation at the point $a_{j}$, then we have

$$
\bar{f}_{i}(\lambda)=(-1)^{n}\left(\operatorname{det} \gamma\left(a_{j}\right)\right)^{-1} \lambda^{n} f_{j}\left(\frac{1}{\lambda}\right) .
$$


d) The coefficients of the characteristic equation fulfil the relations

$$
\begin{aligned}
& \tilde{b}_{n-k}=(-1)^{n}\left(\operatorname{det} \gamma\left(a_{j}\right)\right)^{-1} b_{k} \quad(k=1, \ldots, n+1), \\
& b_{0}=(-1)^{n}, \quad b_{n}=\operatorname{det} \gamma\left(a_{j}\right) .
\end{aligned}
$$

e) If $\operatorname{det}(\mathbf{A}+\mathbf{B})$ is either real or purely imaginary, then

$$
\operatorname{det} \mathrm{g}= \pm 1, \quad \operatorname{det} \gamma\left(a_{j}\right)= \pm 1 \text {. }
$$

Proof: First we have $(\mathbf{A}+\mathbf{B})=(\overline{\mathbf{A}-\mathbf{B}})$. This implies $\operatorname{det} \mathbf{g}=(\operatorname{det}(\dot{\mathbf{A}}+\mathbf{B}))^{-1}$ $\times \operatorname{det}(\mathbf{A}-\mathbf{B})=(\operatorname{det}(\overline{\mathbf{A}-\mathbf{B}}))^{-1} \operatorname{det}(\mathbf{A}-\mathbf{B})$ and $|\operatorname{det} g|=1,\left|\operatorname{det} \gamma\left(a_{j}\right)\right|=\mathbf{1}(j=1$, $\ldots, m)$. Hencefollows $\left.g^{-1}=\left((\mathbf{A}+\mathbf{B})^{-1}(\mathbf{A}-\mathbf{B})\right)^{-1}=(\overline{(\mathbf{A}-\mathbf{B}})^{-1}(\overline{\mathbf{A}+\mathbf{B}})\right)^{-1}=(\overline{\mathbf{A}+\mathbf{B}})^{-1}$ $\times(\overline{\mathbf{A}-\mathbf{B}})=\overline{\mathbf{g}}, \operatorname{det} \gamma^{-1}\left(a_{j}\right)=\operatorname{det}\left[\left(\mathbf{g}^{-1}\left(a_{j}+0\right) \cdot \mathbf{g}\left(a_{j}^{\prime}-0\right)\right)^{-1}\right]=\operatorname{det}\left[\mathrm{g}^{-1}\left(a_{j}-\mathbf{0}\right)\right.$ $\left.\times \mathbf{g}\left(a_{j}+0\right)\right]=\operatorname{det} \overline{\left[\mathbf{g}\left(a_{j}-0\right) \mathbf{g}\left(a_{j}+0\right)^{-1}\right.}=\operatorname{det}\left[\overline{\left.\mathrm{g}\left(a_{j}+0\right)^{-1} \mathbf{g}\left(a_{j}-0\right)\right]}=\operatorname{det} \overline{\gamma\left(a_{j}\right)}\right.$. Because the characteristic equations of the matrix products SD and DS coincide (S, D. - arbitrary $(n, n)$-matrices), we have

$$
\begin{aligned}
\bar{f}_{j}(\lambda) & =\operatorname{det}\left[\overline{\mathbf{g}^{-1}\left(a_{j}+0\right) \mathbf{g}\left(a_{j}-0\right)}-\lambda \mathbf{E}\right]=\operatorname{det}\left[\mathbf{g}\left(\dot{a}_{j}+0\right) \mathrm{g}\left(a_{j}-0\right)^{-1}-\lambda \mathbf{E}\right] \\
& =\lambda^{n} \operatorname{det}\left[\frac{1}{\lambda} \mathbf{g}\left(a_{j}+0\right) \mathbf{g}\left(a_{j}-0\right)^{-1}-\mathbf{E}\right] \\
& =\lambda^{n} \operatorname{det}\left\{g\left(a_{j}+0\right) \mathbf{g}\left(a_{j}-0\right)^{-1}\left[\frac{1}{\lambda} \mathbf{E}-\mathbf{g}\left(a_{j}-0\right) g\left(a_{j}+0\right)^{-1}\right]\right\} \\
& =\lambda^{n}\left(\operatorname{det} \gamma\left(a_{j}\right)\right)^{-1}(-1)^{n} \operatorname{det}\left[\mathbf{g}\left(a_{j}+0\right)^{-1} \mathbf{g}\left(a_{j}-0\right)-\frac{1}{\lambda} \mathbf{E}\right] \\
& =\lambda^{n}\left(\operatorname{det} \gamma\left(a_{j}\right)\right)^{-1}(-1)^{n} f_{j}\left(\frac{1}{\lambda}\right) .
\end{aligned}
$$

This also implies proposition $\mathrm{d}$. Let $\operatorname{det}(\mathbf{A}+\mathbf{B})$ be real or purely imaginary. Then the same is true for $\operatorname{det}(\mathbf{A}-\mathbf{B})=\operatorname{det}(\overline{\mathbf{A}+\mathbf{B}})$. From this, $\operatorname{det} \mathbf{g}= \pm 1, \operatorname{det} \gamma\left(a_{j}\right)$ $= \pm 1$ is easily seen. The lemnia is completely proved

Furthermore, the following lemma holds.

Lemma.2: Let $|\operatorname{det} \mathrm{g}(t)|$ be a continuous function on $L$. Then the numbers $\varrho_{l}{ }^{j}$ fulfil the relations

$$
\operatorname{Im}\left(\sum_{l=1}^{n} \varrho_{l}^{j}\right)=0 \quad(j=1, \ldots, m) .
$$

Proof: We start from (7.8). It is a result of Vekua's theory that the expression in brackets in (7.8) is a continuous non-vanishing function on $L$. By setting $\varrho_{l}^{j}=\alpha_{l}^{j}$. $+i \beta_{l}^{j}$, the bracket of $(7.8)$ is of the for $m$

$$
\begin{aligned}
& \arg \frac{\operatorname{det} g(t)}{\prod_{j=1}^{m} \prod_{l=1}^{n}\left(t-z_{0}\right)^{\left(\alpha_{l}{ }^{i}+i \beta_{l}{ }^{i}\right)}} \\
& =\arg \left\{\operatorname{det} g(t) \prod_{j=1}^{m} \prod_{l=1}^{n}\left[\left|t-z_{0}\right|^{-\left(\alpha_{l}^{y}+i \beta_{l}^{y}\right)} \mathrm{e}^{\beta_{l} \operatorname{sarg}\left(t-z_{0}\right)} \mathrm{e}^{-i \alpha_{t} \operatorname{sarg}\left(t-z_{0}\right)}\right]\right\} .
\end{aligned}
$$

If we consider the absolute value of (7.10), the formula (7.9) follows 'immediately from the continuity 
Corollariés: 1. Lemma 2 is true, especially, if the assumptions of Lemma 1 are fulfilled.

2. Let the assumptions of Lemma 2 be satisfied. Then the formula (7.8) can be simplified to

$$
x_{h}=\frac{1}{2 \pi}\left[\arg \frac{\operatorname{det} \mathrm{g}(t)}{\prod_{j=1}^{m} \prod_{l=1}^{n}\left(t-z_{0}\right)^{\alpha^{2}}}\right]_{L}
$$

where $a_{l}^{j}=\operatorname{Re} \varrho_{i}^{j}$.

The first proposition follows immediately from Lemma 1.d. Obviously, both the first and second factor in the square brackets of (7.10) do not change the argument by circulation on $\mathrm{L}$. This implies the second proposition

Furthermore, we remark that under the assumptions of Lemma 2 (or Lemma 1) it is sufficient for the calculation of the index $x_{h}$ to determine the quantities arg $\left(\lambda_{k}{ }^{j}\right)$. of the roots of the characteristic equations. This follows from

$$
\arg \lambda_{l}^{j}=\mathrm{e}^{2 \pi i a_{i}{ }^{\prime}} \text {. }
$$

\section{\$ 8 Dominant part of (5.10). The matrix $g(t)$}

Henceforth, let us assume again that $L \in C^{2, \beta}$. In consequence of the results in $\S 4$, the integral equation (5.10) satisfies the assumptions of Theorem 7.1. By using (4.2) and (4.3), one can separate the dominant part from the system (5.10). This dominant part is

$$
\mathbf{A}(t) \boldsymbol{\Phi}(t)+\frac{\mathbf{B}(t)}{\pi i} \int_{L} \frac{\Phi(\tau)}{\tau-t} d \tau
$$

where

$A(t)$

$$
B(t)
$$

$t$ from

$\left[\begin{array}{llll}0 & 0 & 0 & 0 \\ 0 & 0 & 0 & 0 \\ 1 & 0 & 1 & 0 \\ 0 & 1 & 0 & 1\end{array}\right]$

$$
i\left[\begin{array}{rccc}
-a_{0} & 0 & a_{1} & 0 \\
0 & -a_{0} & 0 & a_{1} \\
0 & c_{0} & 0 & -c_{1} \\
-c_{0} & 0 & c_{1} & 0
\end{array}\right]
$$

$\left[\begin{array}{cccc}0 & 0 & -n_{2} & n_{1} \\ n_{2} & -n_{1} & 0 & 0 \\ 0 & 0 & 0 & 0 \\ n_{1} & n_{2} & n_{1} & n_{2}\end{array}\right]$

$i\left[\begin{array}{cccc}0 & 0 & c_{1} n_{1} & c_{1} n_{2} \\ c_{0} n_{1} & -c_{0} n_{2} & 0 & 0 \\ -a_{0} n_{1} & -a_{0} n_{2} & a_{1} n_{1} & a_{1} n_{2} \\ -c_{0} n_{2} & c_{0} n_{1} & c_{1} n_{2} & -c_{1} n_{1}\end{array}\right]$

$L_{2}$

$\left[\begin{array}{cccc}0 & 0 & 0 & 0 \\ 0 & 0 & 0 & 0 \\ 0 & 0 & 0 & 0 \\ n_{1} & n_{2} & n_{1} & n_{2}\end{array}\right]$

$i\left[\begin{array}{cccc}0 & 0 & -a_{1} n_{2} & a_{1} n_{1} \\ -a_{0} n_{2} & a_{0} n_{1} & 0 & 0 \\ -a_{0} n_{1} & -a_{0} n_{2} & a_{1} n_{1} & a_{1} n_{2} \\ -c_{0} n_{2} & c_{0} n_{1} & c_{1} n_{2} & -c_{1} n_{1}\end{array}\right]$

$\left[\begin{array}{rrrr}0 & 0 & 0 & 0 \\ 0 & 0 & 0 & 0 \\ 0 & 0 & 0 & 0 \\ -n_{2} & n_{1} & -n_{2} & n_{1}\end{array}\right]$

$i\left[\begin{array}{cccl}0 & 0 & a_{1} n_{1} & a_{1} n_{2} \\ a_{0} n_{1} & a_{0} n_{2} & 0 & 0 \\ a_{0} n_{2} & -a_{0} n_{1} & -a_{1} n_{2} & a_{1} n_{1} \\ -c_{0} n_{1} & -c_{0} n_{2} & c_{1} n_{1} & c_{1} n_{2}\end{array}\right]$ 


$\left[\begin{array}{cccc}0 & 0 & n_{1} & n_{2} \\ -n_{1} & -n_{2} & 0 & 0 \\ 0 & 0 & 0 & 0 \\ -n_{2} & n_{1} & -n_{2} & n_{1}\end{array}\right]$

$$
i\left[\begin{array}{cccc}
0 & 0 & c_{1} n_{2} & -c_{1} n_{1} \\
c_{0} n_{2} & -c_{0} n_{1} & 0 & 0 \\
a_{0} n_{2} & -a_{0} n_{1} & -a_{1} n_{2} & a_{1} n_{1} \\
-c_{0} n_{1} & -c_{0} n_{2} & c_{1} n_{1} & c_{1} n_{2}
\end{array}\right]
$$

$\left[\begin{array}{llll}0 & 0 & 0 & 0 \\ 0 & 0 & 0 & 0 \\ 0 & 0 & 0 & 0 \\ 0 & 0 & 0 & 0\end{array}\right]$

$i\left[\begin{array}{cccc}a_{0} & 0 & 0 & 0 \\ 0 & a_{0} & 0 & 0 \\ 0 & 0 & a_{1} & 0 \\ 0 & 0 & 0 & a_{1}\end{array}\right]$

$L_{6}$

$\left[\begin{array}{rrrr}-1 & 0 & 0 & 0 \\ 0 & -1 & 0 & 0 \\ 0 & 0 & 1 & 0 \\ 0 & 0 & 0 & 1\end{array}\right]$

$i\left[\begin{array}{cccc}0 & -c_{0} & 0 & 0 \\ c_{0-} & 0 & 0 & 0 \\ 0 & 0 & 0 & -c_{1 !} \\ 0 & 0 & c_{1} & 0\end{array}\right]$

$L_{7}$

$\left[\begin{array}{cccc}0 & 0 & 0 & 0 \\ n_{2} & -n_{1} & 0 & 0 \\ 0 & 0 & 0 & 0 \\ 0 & 0 & -n_{2} & n_{1}\end{array}\right]$

$i\left[\begin{array}{llll}a_{0} n_{1} & a_{0} n_{2} & 0 & 0 \\ c_{0} n_{1} & c_{0} n_{2} & 0 & 0 \\ 0 & 0 & a_{1} n_{1} & a_{1} n_{2} \\ 0 & 0 & c_{1} n_{1} & c_{1} n_{2}\end{array}\right]$

$\left[\begin{array}{cccc}0 & 0 & 0 & 0 \\ -n_{1} & -n_{2} & 0 & 0 \\ 0 & 0 & 0 & 0 \\ 0 & 0 & n_{1} & n_{2}\end{array}\right]$

$i\left[\begin{array}{cccc}-a_{0} n_{2} & a_{0} n_{1} & 0 & 0 \\ c_{0} n_{2} & -c_{0} n_{1} & 0 & -0 \\ 0 & 0 & -a_{1} n_{2} & a_{1} n_{1} \\ 0 & 0 & c_{1} n_{2} & -c_{1} n_{1}\end{array}\right]$

and

$$
a_{l}=\frac{\lambda_{l}+3 \mu_{l}}{2 \mu_{l}\left(\lambda_{l}+2 \mu_{l}\right)}, \quad c_{l}=\frac{\mu_{l}}{\lambda_{l}+2 \mu_{l}}:\left(\lambda_{l}, \mu_{l}>0: l=0,1\right)
$$

$n_{1}, n_{2}$ are the direction cosines of the normal vector $\mathbf{n}$ at the point $t \in L$ :

It is immediately seen, that the system (5.10) with its dominant part (8.1) satisfies the assumption of Lemma 7.1. As a consequence of Lemma 7.1a, (5.10). is of regular type. The assumption of Lemma $7.1 \mathrm{e}$ is additionally true. Using the multiplication theorem for determinants, one obtains

$$
\operatorname{det} \mathbf{g}(t)= \begin{cases}11 & \text { for } t \in L_{1} \cup L_{6} \cup L_{7} \cup L_{8} \cup L_{9} \\ -1 & \text { for } t \in L_{2} \cup L_{3} \cup L_{4} \cup L_{5} .\end{cases}
$$

In the sequel, the explicit form of the matrices $\mathrm{g}(t)$ is also needed. The results of the calculations are given in the following formulas.

With the abbreviations

$$
\begin{aligned}
& A=\left(a_{0} c_{1}-a_{1} c_{0}\right), \quad B=a_{0}^{2}-a_{1}^{2}, \quad C_{1}=a_{0}+a_{1}, \\
& D_{l}=1+c_{l}{ }^{2}: \quad E_{l}=1-c_{l}^{2}, \quad F=\dot{c}_{0}+c_{1}, \quad G=c_{0} c_{1}, \quad(l=0,1),
\end{aligned}
$$

one gets

$$
\begin{aligned}
& \mathbf{g}(t)=-\frac{1}{N}\left[\begin{array}{llll}
-A^{2}+B & -2 i a_{1} A & -2 a_{1} C & -2 i a_{1} A \\
2 i a_{1} A & -A^{2}+B & 2 i a_{1} A & -2 a_{1} C \\
-2 a_{0} C & -2 i a_{0} A & -A^{2}-B & -2 i a_{0} A \\
2 i a_{A} A & -2 a_{0} C & 2 i a_{0} A & -A^{2}-B
\end{array}\right] \text { for } t \in L_{1},(8.7 \mathrm{a}) \\
& N=C^{2}-A^{2} \text {; }
\end{aligned}
$$




$$
\begin{aligned}
& \mathrm{g}(\dot{t})=\frac{1}{M}\left\{\begin{array}{llll}
0 & a_{1} c_{0} & a_{1} F n_{1} n_{2} & a_{1}\left(c_{0} n_{\dot{2}}^{2}-c_{1} n_{1}{ }^{2}\right) \\
-a_{1} c_{0} & 0 & a_{1}\left(c_{1} n_{2}^{2}-c_{0} n_{1}^{2}\right)-a_{1} F n_{1} n_{2} \\
-a_{0} F n_{1} n_{2} & a_{0}\left(c_{0} n_{1}^{2}-c_{1} n_{2}{ }^{2}\right) & 0 & -a_{0} c_{1} \\
a_{0}\left(c_{1} n_{1}{ }^{2}-c_{0} n_{2}{ }^{2}\right) & a_{0} F n_{1} n_{2} & a_{0} c_{1} & 0
\end{array}\right] \\
& +i\left[\begin{array}{lll}
a_{1} D_{0}+a_{0} E_{1}\left(n_{2}^{2}-n_{1}^{2}\right) & -2 a_{0} E_{1} n_{1} n_{2} & 2 a_{1}\left(n_{1}^{2}-G n_{2}^{2}\right) \\
-2 a_{0} E_{1} n_{1} n_{2} & a_{1} D_{0}+a_{0} E_{1}\left(n_{1}^{2}-n_{2}^{2}\right) & 2 a_{1}(1+G) n_{1} n_{2} \\
2 a_{0}\left(n_{1}^{2}-G n_{2}^{2}\right) & 2 a_{0}(1+G) n_{1} n_{2} & a_{0} D_{1}+a_{1} E_{0}\left(n_{2}^{2}-n_{1}^{2}\right) \\
2 a_{0}(1+G) n_{1} n_{2} & 2 a_{0}\left(n_{2}^{2}-G n_{1}^{2}\right) & -2 a_{1} E_{0} n_{1} n_{2}
\end{array}\right. \\
& \left.\left.\begin{array}{l}
2 a_{1}(1+G) n_{1} n_{2} \\
2 a_{1}\left(n_{2}^{2}-G n_{1}^{2}\right) \\
-2 a_{1} E_{0} n_{1} n_{2} \\
a_{0} D_{1}+a_{1} E_{0}\left(n_{1}^{2}-n_{2}^{2}\right)
\end{array}\right]\right\} \text { for } t \in L_{2}
\end{aligned}
$$

$$
M=i\left(a_{0} E_{1}+a_{1} E_{0}\right)
$$

$g(t)=\frac{1}{C}$

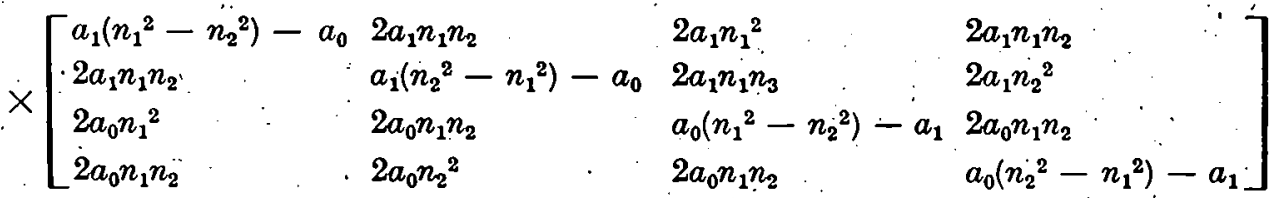
for $t \in L_{3}$;

$\mathrm{g}(t)=\frac{1}{C} ;$

$\times\left[\begin{array}{llll}a_{1}\left(n_{2}^{2}-n_{1}^{2}\right)-a_{0} & -2 a_{1} n_{1} n_{2} & 2 a_{1} n_{2}^{2} & -2 a_{1} n_{1} n_{2} \\ -2 a_{1} n_{1} n_{2} & a_{1}\left(n_{1}^{2}-n_{2}^{2}\right)-a_{0}-2 a_{1} n_{1} n_{2} & 2 a_{1} n_{1}^{2} \\ 2 a_{0} n_{2}^{2} & -2 a_{0} n_{1} n_{2} & a_{0}\left(n_{2}^{2}-n_{1}\right)-a_{1}-2 a_{0} n_{1} n_{2} \\ -2 a_{0} n_{1} n_{2} & 2 a_{0} n_{2}^{2} & -2 a_{0} n_{1} n_{2} & a_{0}\left(n_{1}^{2}-n_{2}^{2}\right)-a_{1}\end{array}\right]$ for . $t \in L_{4}$;

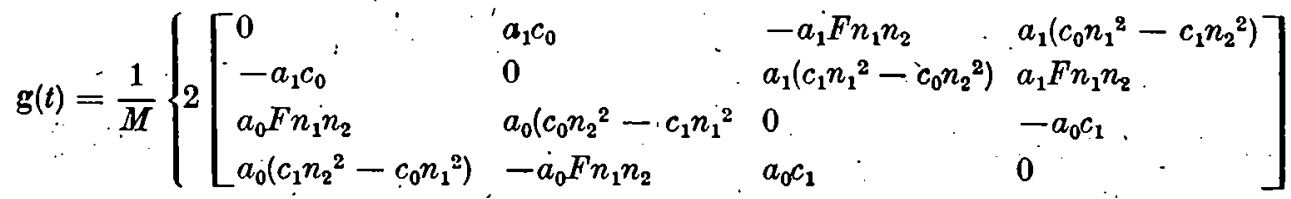

$+i\left[\begin{array}{lll}a_{1} D_{0}+a_{0} \dot{E}_{1}\left(n_{1}{ }^{1}-n_{2}{ }^{2}\right) & 2 a_{0} E_{1} n_{1} n_{2} & 2 a_{1}\left(n_{2}{ }^{2}-G n_{1}{ }^{2}\right) \\ 2 a_{0} E_{1} n_{1} n_{2} & a_{1} D_{0}+a_{0} E_{1}\left(n_{2}{ }^{2}-n_{1}{ }^{2}\right) & -2 a_{1}(1+G)^{\prime} n_{1} n_{2} \\ 2 a_{0}\left(n_{2}{ }^{2}-G n_{1}{ }^{2}\right) & -2 a_{0}(1+G) n_{1} n_{2}: & a_{0} D_{1}+a_{1} E_{0}\left(n_{1}{ }^{2}-n_{2}{ }^{2}\right) \\ -2 a_{0}(1+G) n_{1} n_{2} & 2 a_{0}\left(n_{1}{ }^{2}-G n_{2}{ }^{2}\right) & 2 a_{1} E_{0} n_{1} n_{2} .\end{array}\right.$

$\left.\left.\begin{array}{l}-2 a_{1}(1+G) n_{1} n_{2} \\ 2 a_{1}\left(n_{1}-G n_{2}{ }^{2}\right) \\ 2 a_{1} E_{0} n_{1} n_{2} \\ a_{0} D_{1}+a_{1} E_{0}\left(n_{2}{ }^{2}-n_{1}{ }^{2}\right)\end{array}\right]\right\}$ for $t \in L_{5}$ 


$$
\begin{aligned}
& g(t)=\left[\begin{array}{rrrr}
-1 & 0 & 0 & 0 \\
0 & -1 & 0 & 0 \\
0 & 0 & -1 & 0 \\
0 & 0 & 0 & -1
\end{array}\right] \text { for } t \in L_{6} \\
& \mathbf{g}(t)=\left[\begin{array}{llll}
\frac{1+c_{0}^{2}}{1-c_{0}^{2}} & \frac{-2 i c_{0}}{1-c_{0}^{2}} & 0 & 0 \\
\frac{2 i c_{0}}{1-c_{0}^{2}} & \frac{1+c_{0}^{2}}{1-c_{0}^{2}} & 0 & 0 \\
0 & 0 & \frac{1+c_{1}^{2}}{1-c_{1}^{2}} & \frac{2 i c_{1}}{1-c_{1}^{2}} \\
0 & 0 & \frac{-2 i c_{1}}{1-c_{1}^{2}} & \frac{1+c_{1}^{2}}{1-c_{1}^{2}}
\end{array}\right] \text { for } t \in L_{7}
\end{aligned}
$$

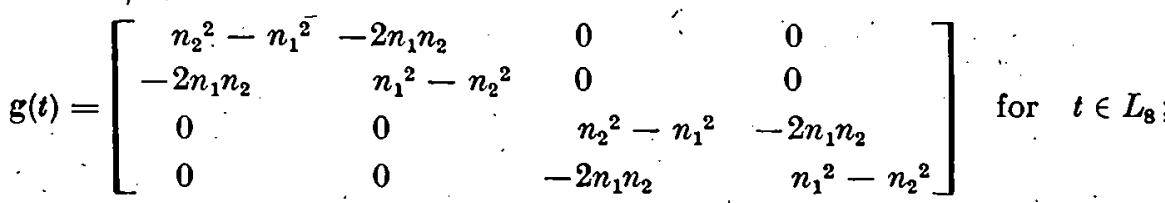

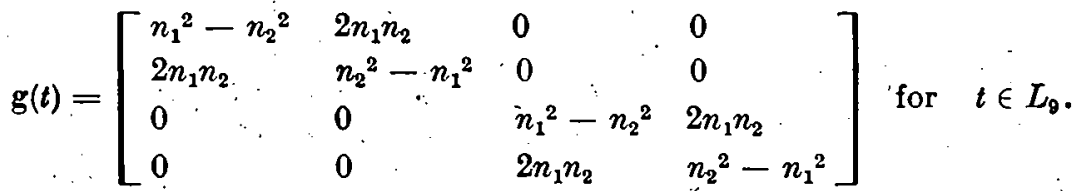

\section{\$9 Determinant and trace of $\gamma^{j}$}

For the analysis of index of the system (5.10), the roots $\lambda_{1}{ }^{j}, \lambda_{2}{ }^{j}, \ldots, \lambda_{4}{ }^{j}$ of the characteristic equations (7.6) of matrices $\gamma^{i}(7.5)$ have to be determined. Because the nodes $a_{j}$ of the problem $C^{*}$ can be of 72 different type, each of those possibilities must be discussed.

In virtue of Lemma $7.1 \mathrm{~b}$ we have

$$
\begin{aligned}
& \gamma^{-1}\left(a_{j}\right)=\mathbf{g}^{-1}\left(a_{i}-0\right) \mathbf{g}\left(a_{j}+0\right)=\overline{\mathbf{g}\left(a_{j}-0\right)} \mathbf{g}\left(a_{j}+0\right) \\
& \gamma\left(a_{j}\right)=\mathrm{g}^{-1}\left(a_{j}+0\right) \mathbf{g}\left(a_{j}-0\right)=\overline{\mathbf{g}\left(a_{j}+0\right)} \mathbf{g}\left(a_{j}-0\right) .
\end{aligned}
$$

-Thus, the coefficients of the characteristic equations of $\gamma^{-1^{\prime}}\left(a_{j}\right)$ and $\gamma\left(a_{j}\right)$ are conjugate complex.

The following analysis shows that the characteristic equations are real for all possible combinations. Consequently, the characteristic equations at a node $a_{i}$ of the type $L_{j}-L_{k}$ are the same as for the type $L_{k}-L_{j}(j, k=1,2, \ldots, 9)$. Hence it is sufficient to consider 36 different type of characteristic equations.

The problem of finding the characteristic equations is equivalent to the determination of the invariants $b_{4}{ }^{j}=\operatorname{det} \gamma^{j}, b_{1}{ }^{j}=-\operatorname{tr}\left(\gamma^{j}\right)$ and the second invariant $b_{2}{ }^{j}$. The remaining invariants can be obtained by u'sing Lemma $7.1 \mathrm{~d}$.

The values of $b_{4}{ }^{3}$ follow immèdiately from (8.5).

More difficult is the analysis of the traces. For this purpose, the diagonal elements of the matrices $\gamma^{j}$ must be calculated. In order to distinguish the matrices $\gamma^{j}$ for nodes $a_{j}$ of different 
kind, these matrices are designated by $\gamma_{(l-k)}^{j}$, which means the matrix $\gamma^{j}$ at the node $a_{j}$ of

type $L_{l}-L_{k}(l, k=1,2, \ldots, 9 ; l \neq k)$.
$\quad$ First, let us consider the case $L_{1}-L_{2}$. Let $\gamma_{(1-2)}^{j}=-\frac{1}{N M} \cdot\left[M_{i k}\right]_{i, k-1, \ldots, 4}$ (the constants $N, M$ are defined by $(8.7 \mathrm{a})$ and $(8.7 \mathrm{~b}))$. Then one obtains for the diagonal elements

$$
M_{k k}=P_{k k}+Q_{k k} n_{1}^{2}+R_{k k} n_{2}^{2}+S_{k k} n_{1} n_{2} \quad(k=1, \ldots, 4)
$$

and

$$
\begin{aligned}
P_{11}=-i a_{1}\left[\left(A^{2}-B\right) D_{0}+4 a_{1} c_{0} A\right]=P_{22}, \quad Q_{11}=i a_{0}\left[E_{1}\left(A^{2}-B\right)-4 a_{1} C+4 a_{1} c_{1} A\right]=R_{22} \\
Q_{22}=-i a_{0}\left[E_{1}\left(A^{2}-B\right)+4 a_{1} c_{0} A-4 a_{1} C G\right]=R_{11} \\
S_{11}=4 a_{0} a_{1}\left[A E_{1}+C F-A(1+G)\right]=-S_{22} \\
P_{33}=-i a_{0}\left[\left(A^{2}+B\right) D_{1}-4 a_{0} c_{1} A\right]=P_{44} \\
Q_{33}=-i a_{1}\left[4 a_{0} C+4 a_{0} c_{0} A-\left(A^{2}+B\right) E_{0}\right]=R_{44}, \\
Q_{41}=i a_{1}\left[4 a_{0} c_{1} A+4 a_{0} C G-\left(A^{2}+B\right) E_{0}\right]=R_{33} \\
S_{33}=4 a_{0} a_{1}\left[-C F-A(1+G)+A E_{0}\right]=-S_{44}
\end{aligned}
$$

Taking into account (8.6), one can show

$$
-N M \operatorname{tr}\left(\underline{\gamma}_{(1-2)}^{j}\right)=\sum_{k=1}^{4} M_{k k}=\sum_{k=1}^{4}\left(P_{k k}+Q_{k k}\right)=-2 N M ;
$$

hence follows

$$
\operatorname{tr}\left(\gamma_{(1-2)}^{j}\right)=2 \text {. }
$$

Let us consider the case $L_{1}-L_{3}$. Setting

$$
\gamma_{(1-3)}^{j}=-\frac{1}{N C}\left[L_{i k}\right]_{i, k-1, \ldots, 4}
$$

and

$$
L_{k k}=P_{k k}+Q_{k k} n_{1}^{2}+R_{k k} n_{2}^{2}+S_{k k} n_{1} n_{2} \quad(k=1, \ldots, 4),
$$

we get

$$
\begin{aligned}
& P_{11}=a_{0}\left(A^{2}-B\right)=P_{22}, \quad P_{33}=a_{1}\left(A^{2}+B\right)=P_{44}, \\
& Q_{11}=a_{1}\left(B-A^{2}\right)-4 a_{0} a_{1} C=R_{22}, \quad Q_{33}=-a_{0}\left(A^{2}+B\right)-4 a_{0} a_{1} C=R_{44}, \\
& R_{11}=a_{1}\left(A^{2}-B\right)=Q_{22}, \quad R_{33}=a_{0}\left(A^{2}+B\right)=Q_{44} ; \\
& S_{11}=4 i a_{1}^{2} A+4 i a_{0} a_{1} A=-S_{22}, \quad S_{33}=4 i a_{0} a_{1} A+4 i a_{0}^{2} A=-S_{44} .
\end{aligned}
$$

Therefore $-N C \operatorname{tr}\left(\gamma_{(1-3)}^{j}\right)=\sum_{k=1}^{t} L_{k k}=-2 N C$, this implies

$$
\operatorname{tr}\left(\gamma_{(1-3)}^{j}\right)=2
$$

The same result is obtained for the cases $\dot{L}_{1}-L_{4}$ and $L_{1}-L_{5}$ :

$$
\operatorname{tr}\left(\gamma_{(1-4)}^{j}\right)=\operatorname{tr}\left(\gamma_{(1-5)}^{j}\right)=2 .
$$

It it not difficult to get

$$
\operatorname{tr}\left(\gamma_{(1-6)}^{j}\right)=-4 \frac{A^{2}}{C^{2}-A^{2}}=\omega_{1} .
$$


With $\gamma_{(1-7)}^{j}=-\frac{1}{N}\left[\dot{H_{i k}}\right]_{i, k=1, \ldots, 4}$ one obtains

$$
\begin{gathered}
H_{11}=\frac{1}{E_{0}}\left[D_{0}\left(B-A^{2}\right)-4 a_{1} c_{0} A\right]=H_{22}, \quad H_{33}=\frac{1}{E_{1}}\left[-D_{1}\left(A^{2}+B\right)+4 a_{n} c_{1} A\right]^{\prime}=H_{44}, \\
\therefore \quad \operatorname{tr}\left(\gamma_{(1-7)}^{j}\right)=\frac{-4}{E_{0} E_{1}} \frac{\left(a_{0} c_{0} E_{1}-a_{1} c_{1} E_{0}\right)^{2}}{C^{2}-A^{2}}=\omega_{2} .
\end{gathered}
$$

The calculated values for $\operatorname{tr}\left(\gamma_{(l-k)}^{j}(l-k)\right)$ and $\operatorname{det}\left(\gamma_{(l-k)}^{j}\right)$ are collected in the fol-

\begin{tabular}{|c|c|c|c|c|c|c|c|}
\hline type $l-k$ & & $\operatorname{det}\left(\gamma_{(l-k)}^{j}\right)$ & $\operatorname{tr}\left(\gamma_{(l-k)}^{j}\right)$ & type $l-k$ & & $\operatorname{det}\left(\gamma_{(l-k)}^{j}\right)$ & $\operatorname{tr}\left(\gamma_{(l-k)}^{j}\right)$ \\
\hline $1-2$ & & -1. & 2 & $3-7$ & & -1 & $\omega_{5}$ \\
\hline $1-3$ & . & -1 & 2 & $3-8$ & & -1 & 2 \\
\hline $1-4$ & & -1 & 2 & $3-9$ & & -1 & -2 \\
\hline $1-5$ & & -1 & 2 & $4-5$ & & 1 & 0 \\
\hline $1-6$ & & 1. & $\omega_{1}$ & $4-6$ & & -1 & 2 \\
\hline $1-7$ & & 1 & $\omega_{2}$ & $4-7$ & & -1 & $\omega_{5}$ \\
\hline $1-8$ & & 1 & 0 & $4-8$ & & $-i$ & -2 \\
\hline $1-9$ & & 1 & 0 & , $4-9$ & & -1 & 2 \\
\hline $2-3$ & & 1 & 0 & $5-6$ & & -1 & $\omega_{4}$ \\
\hline $2-4$ & & 1 & $\omega_{3}$ & $5-7$ & & -1 & 2 \\
\hline $2-5$ & & 1 & 0 & $5-8$ & & -1 & $\cdot 2$ \\
\hline $2-6$ & & -1 & $\omega_{4}$ & $5-9$ & & $\therefore 1$ & -2 \\
\hline $2-7$ & & -1 & 2 & $6-7$ & & 1 & $\omega_{0}$ \\
\hline $2-8$ & . & -1 & -2 & $6-8$ & & 1 & 0 \\
\hline $2-9$ & , & $=1$ & 2 & $6-9$ & & 1 & 0 \\
\hline $3-4$ & & 1 & 0 & $7-8$ & & 1 & 0 \\
\hline $3-5$ & & 1 & $\omega_{3}$ & $7-9$ & & 1 & 0 \\
\hline $3-6$ & & -1 & 2 & $8-9$ & & 1 & -4 \\
\hline
\end{tabular}
lowing table:

Here, $\omega_{1}$ and $\omega_{2}$ are given by (9.5), and (9.6), respectively. Furthermoré, we have

$$
\begin{aligned}
& \omega_{3}=-\frac{4 a_{0} a_{1} F^{2}}{C\left(a_{0} E_{1}+a_{1} E_{0}\right)}, \\
& \omega_{4}=-\frac{2\left(a_{1} D_{0}+a_{0} D_{1}\right)}{a_{1} E_{0}+a_{0} E_{1}}, \\
& \omega_{5}=-2 \frac{E_{0} E_{1} C+2\left(a_{0} c_{0}^{2} E_{1}+a_{1} c_{1}{ }^{2} E_{0}\right)}{E_{0} E_{1} C}, \\
& \omega_{6}=\frac{-2}{E_{0} E_{1}}\left(D_{0} E_{1}+D_{1} E_{0}\right):
\end{aligned}
$$

Because of $0<c_{k}=\frac{\mu_{k}}{\lambda_{k}+2 \mu_{k}}<\frac{1}{2}$ and $a_{k}>0(k=0,1)$ we have

$0 \geqq \omega_{1}=-4 \frac{\left(a_{0} c_{1}-a_{1} c_{0}\right)^{2}}{\left(a_{0}+a_{1}\right)^{2}-\left(a_{0} c_{1}-a_{1} c_{0}\right)^{2}}>-4 \frac{1 / 4\left(a_{0}+a_{1}\right)^{2}}{3 / 4\left(a_{0}+a_{1}\right)^{2}}=-\frac{4}{3}$. 
Assuming without loss of generality that $E_{0} \leqq E_{1}$, one obtains

$$
\begin{aligned}
0 \geqq & \omega_{2}=-4 \frac{a_{0}^{2} c_{0}^{2} E_{1}^{2}+a_{1}^{2} c_{1}{ }^{2} E_{0}^{2}-2 a_{0} a_{1} c_{0} c_{1} E_{0} E_{1}}{\left(a_{0}+a_{1}\right)^{2}-\left(a_{0} c_{1}-a_{1} c_{0}\right)^{2}} \frac{1}{E_{0} E_{1}}>-4 \frac{E_{1}}{E_{0}} \\
& \times \frac{a_{0}{ }^{2} c_{0}^{2}+a_{1}{ }^{2} c_{1}^{2}}{\left(a_{0}+a_{1}\right)^{2}-\left(a_{0} c_{1}-a_{1} c_{0}\right)^{2}}>-4 \frac{1}{3 / 4} \frac{1 / 4\left(a_{0}+a_{1}\right)^{2}}{3 / 4\left(a_{0}+a_{1}\right)^{2}}=-\frac{16}{9}
\end{aligned}
$$

Similarly, taking into account $\frac{2}{3} E_{k}>2 c_{k}^{2}(k=0,1)$, we get

$$
\begin{aligned}
0 & >\omega_{3}=-4 \frac{a_{0} a_{1}\left(c_{0}+c_{1}\right)^{2}}{\left(a_{0}+a_{1}\right)\left(a_{0} E_{1}+a_{1} E_{0}\right)} \geqq-\frac{\left(a_{0}+a_{1}\right)\left(c_{0}+c_{1}\right)^{2}}{\left(a_{0} E_{1}+a_{1} E_{0}\right)} \\
& >-\frac{\left(c_{0}+c_{1}\right)^{2}}{3 / 4}>-\frac{4}{3},
\end{aligned}
$$

and

$$
\begin{aligned}
& -2>\omega_{4}>-\frac{10}{3} \\
& -2>\omega_{5}>-\frac{10}{3}
\end{aligned}
$$

\section{$\$ 10 \cdot$ Roots $\lambda_{l}$ if the characteristic equations and index $x_{h}$ of the operator $\Omega_{p} \mathscr{A}$}

For determination of the indices $\varkappa_{h}$ by formula (7.8), some additional information concerning the roots $\lambda_{l}{ }^{j}$ of the characteristic equations (7.6) of the system $(5.10)$ with its dominant part (8.1) is necessary. Since the assumptions of Lemmata 7.1 and 7.2 are fulfilled, the indices can be calculated by using the more simple formula (7.11). Hence, the values $\alpha_{l}{ }^{j}=\operatorname{Re} \varrho_{l}{ }^{j}=\arg \lambda_{l}{ }^{j}$ give sufficient information to determine the indices.

A first possible way for the further considerations might be the direct counting of the second invariant $b_{2}{ }^{i}$ of matrices $\gamma\left(a_{j}\right)$. However, this approach is connected with very, extensive calculations, which are not worth while, especially for nodes of the types $(1-2),(2-3),(2-4),(2-5),(1-5)$, and others, for instance. Therefore another less extensive way will be gone. This approach makes use of results obtained in $\S \S 5,6$.

In preparation of the following considerations we prove the following lemma.

Lemma 1: Let 9 be a linear manifold of 4-component vectors on $L$ containing the vectors $\mathbf{w}_{1}, \mathbf{w}_{2}$ of $\S 5$. Let the vectors $\mathbf{h} \in \mathfrak{R}$ satisfy the assumptions of $\$ .2$. Suppose that the homogeneous problem $C^{*}$ has exactly $h$ linearly independent solutions. Consider the linear manifolds

$$
\begin{aligned}
& \mathfrak{M}=\left\{\boldsymbol{\Phi} \in H^{*} / \mathscr{\Phi}=\mathbf{h}, \mathbf{h} \in \mathfrak{N}\right\}, \\
& \mathfrak{M}_{0}=\left\{\boldsymbol{\Phi} \in \underset{\mathcal{N} / \int_{L}}{ } \varphi^{0}(\mathbf{y}) . d s_{\mathbf{y}}=\mathbf{0}\right\}
\end{aligned}
$$

Then we have $\operatorname{dim} \mathfrak{D} \leqq h+s, \operatorname{dim} \mathfrak{M}_{0} \leqq h+s-2$ provided that $s=\operatorname{dim} \mathfrak{N}$.

For proof we remark that the problem $C^{*}$ with contact data in $\mathfrak{R}$ permits, in virtue of linearity of $C^{*}$, no more than $h+s$ linearly independent solutions. The continuation is the rather precise repetition of the proof of Lemma 5.1

For the further considerations concerning the characteristic equation in a given node $a_{j}$ of the type $(l-k)(l, k=1, \ldots, 9 ; l \neq k)$, the following auxiliary problem $C_{(l-k)}^{*}$ is important. 
This problem $C_{(-k)}^{*}$ is defined by the following figure 1 . The auxiliary problem $C_{(l-k)}^{*}$ has 10 nodes $\tilde{a}_{1}, \tilde{a}_{2}, \ldots, \tilde{a}_{10}$, whose normal vector $\mathbf{n}$ is the same as.at the considered node $\tilde{a}_{1}=a_{j}$ of the original problem $C^{*}$. Moreover, the boundary of $\tilde{D}_{1}$ is constructed in such a way that the homogeneous problem $C_{(-k)}^{*}$ does not permit any additional solutions from the tables $2-4$ of $\S 3$. Consequently, the homogeneous problem $C_{(l-k)}^{*}$ has exactly 2 linearly independent solutions,

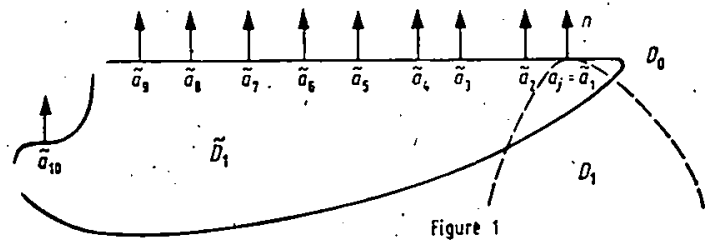

Auxtliery problem (li-k)

if $l$ and $k$ have one of the values $1,2,5$, or 7 . In the remaining cases the homogeneous problem $C_{(-k)}^{*}$ allows only the trivial solution. It is important that the characteristic equations (7.6) at the node $\tilde{a}_{1}=a_{j}$ are one and the same for the problems $C_{(1-k)}^{*}$ and $C^{*}$. Using the formula (9.1), it is easily seen that the characteristic equations at $\tilde{a}_{2}, \tilde{a}_{4}, \ldots, \tilde{a}_{10}$ have conjugate complex coefficients regarding the characteristic equation at $\bar{a}_{1}$, Moreover, we have

$$
\gamma\left(\tilde{a}_{j}\right)=\gamma^{-1}\left(\tilde{a}_{1}\right) \text { for } j=2,4,6,8,10
$$

and

$$
\gamma\left(\tilde{a}_{j}\right)=\gamma\left(\bar{a}_{1}\right) \text { for } j=3,5,7,9 \text {. }
$$

Let $\lambda_{1}, \lambda_{2}, \lambda_{3}, \lambda_{4}$ be the roots of the characteristic equation $f_{1}(\lambda)=\operatorname{det}\left(\gamma\left(\bar{a}_{f}\right)-\lambda E\right)=0$.

Then the roots of the characteristic equation

$$
f_{j}(\lambda)=\bar{f}_{1}(\lambda)=\operatorname{det}\left(\gamma\left(\tilde{a}_{j}\right)-\lambda \mathbf{E}\right)=0 \quad(j=2,4,6,8,10)
$$

are $\bar{\lambda}_{1}, \bar{\lambda}_{2}, \bar{\lambda}_{3}, \bar{\lambda}_{4}$. In virtue of the considerations above, we have in the sense of set theory

$$
\left\{\bar{\lambda}_{1}, \bar{\lambda}_{2}, \bar{\lambda}_{3}, \bar{\lambda}_{4}\right\}=\left\{\lambda_{1}^{-1}, \lambda_{2}^{-1}, \lambda_{3}^{-1}, \lambda_{4}^{-1}\right\} \text {. }
$$

After these preparations, let us consider the characteristic equations $f_{j}(\lambda)=0$. In consequence of Lemma $7.1 \mathrm{~d}$, the second invariant $b_{2}^{j}$ of the matrices $\gamma\left(a_{j}\right)$ satisfies the following relations

$$
\begin{aligned}
& \operatorname{Re} b_{2}^{j}=0 \quad \text { for } \operatorname{det} \gamma\left(a_{j}\right)=-1 \\
& \operatorname{Im} b_{2}^{j}=0 \text { for } \operatorname{det} \gamma\left(a_{j}\right)=1
\end{aligned}
$$

First, the cases with det $\gamma\left(a_{j}\right)=-1$ are studied. From the table in $\$ 9$ one can see the following form of the characteristic equations (using Lemma 7.1 d).

Case 1: $f_{j}(\lambda)=\lambda^{4}-2 \lambda^{3}+i \sigma \lambda^{2}+2 \lambda-1=0$

(for nodes $a_{i}$ of the type $(1-2),(1-3),(1-4),(1-5),(2-7),(2-9),(3-6),(3-8)$, $(4-6),(4-9),(5-7),(5-8))$.

Case 2: $f_{i}(\lambda)=\lambda^{4}+2 \lambda^{3}+i \sigma \lambda^{2}-2 \lambda-1=0$

(for nodes $a_{i}$ of the type $\left.(2-8),(3-9),(4-8),(5-9)\right)$.

Case 3: $\quad f_{j}(\lambda)=\lambda^{4}-\omega_{1} \lambda^{3}+i \sigma \lambda^{2}+\omega_{1} \lambda-1=0$

(for type $(2-6)$ and $(5-6))$.

Case 4: $f_{i}(\lambda)=\lambda^{4}-\omega_{5} \lambda^{3}+i \sigma \lambda^{2}+\omega_{5} \lambda-1=0$

(for type $(3-7)$ and $(\dot{4}-7))$.

Lemma 2: Let $\operatorname{det} \gamma\left(a_{j}\right)=-1$. Then follows $b_{2}{ }^{j}=0$. 
For proof suppose $b_{2}{ }^{j} \neq 0$. Then we have $b_{2}{ }^{j}=i \sigma(\sigma \neq 0$, real). The characteristic equation $f_{j}(\lambda)=0$ is of the form $(10.5),(10.6),(10.7)$ or $(10.8)$, where the considered node $a_{j}$ is supposed to be of the type $l-k$. Let

$$
\lambda_{h}^{j}=r_{h} \mathrm{e}^{2 \pi i \alpha_{h}}, \quad(h=1, \ldots, 4)
$$

be the roots of the characteristic equation. Because of $\sigma \neq 0$ we have $\alpha_{h}^{j} \neq 0, \pm \frac{1}{2} ; r_{h} \neq 0$.

Now we consider the auxiliary problem $C_{(l-k)}^{*}$. Let the corresponding integral equation system be

$$
\Omega_{p} \mathscr{A} \Phi=A_{1} \Omega_{p} \mathrm{w}_{1}+A_{2} \Omega_{p} \mathrm{w}_{2},
$$

which is to be studied in the class $h^{*}$. Hence

$$
-1<\alpha_{h}^{i}<0
$$
and, consequently, the nodes $\tilde{a}_{1}, \ldots, \tilde{a}_{10}$ are nonspecial. Because of the relation $\prod_{h=1}^{4} \lambda_{h}^{j}=-1$,
we have one of the following sums

$$
\sum_{h=1}^{4} \alpha_{h}^{j}=-\frac{1}{2},-\frac{3}{2},-\frac{5}{2},-\frac{7}{2} .
$$

This implies

$$
\sum_{h=1}^{4} \tilde{\alpha}_{h}{ }^{k}=-\frac{1}{2},-\frac{3}{2},-\frac{5}{2},-\frac{7}{2} \quad \text { for } k=1,3,5,7,9
$$

and, because of (10.3),

$\sum_{h=1}^{4} \tilde{\alpha}_{h}^{k}=\sum_{h=1}^{4}\left(-1^{\prime}-\tilde{\alpha}_{h}{ }^{1}\right)=-\frac{7}{2},-\frac{5}{2},-\frac{3}{2},-\frac{1}{2} \quad$ for $k=2,3,6,8,10$.

Taking into account $(* *)$ and $(* * *)$, the indes $*_{n^{*}}$ of $(*)$ is calculated by formula $(7.11)$ as follows:

$$
x_{h^{*}}=\frac{1}{2 \pi}\left[\arg \frac{1}{\prod_{j=1}^{10} \prod_{k=1}^{4}\left(t-z_{0}\right)^{a_{k^{3}}}}\right]_{L}=\frac{1}{2 \pi}\left[\arg \left(t-z_{0}\right)^{20}\right]_{L}=20 .
$$

Hence, the equation $(*)$ permits at least 20 linearly independent solutions $\boldsymbol{\Phi}_{1}, \Phi_{2}, \ldots ; \boldsymbol{\Phi}_{20}$.

On the other hand, $(*)$ is equivalent to

$$
\mathscr{A} \boldsymbol{\Phi}=A_{1} \mathbf{w}_{1}+A_{2} \mathbf{w}_{2}+\mathbf{h},: \mathbf{h} \in \operatorname{ker} \Omega_{p} .
$$

The dimension of the linear space ker $\Omega_{p}$ can be obtained by formula (6.11). For case 1 we get $\operatorname{dim} \operatorname{ker} \boldsymbol{\Omega}_{p}=5$. Since the homogeneous problem $C_{(l-k)}^{*}$ allows at most 2 linearly independent solutions, and because of $\operatorname{dim} \mathfrak{L}\left\{\mathbf{w}_{1}, \mathbf{w}_{2}\right\} \leqq 2$, the use of Lemma 1 shows that the equation $(t)$ permits at most 9 linearly independent solutions.

This contradiction proves the proposition in case 1 . In the case $2,3,4$, the formula (6.11) in plies $\operatorname{dim} \operatorname{ker} \Omega_{p}=15$. Consequently; $(+)$ allow's at most 19 linearly independent solutions, which also contradicts the existence of at least 20 linearly independent solutions of equation $(*)$. The lemma is completely proved

In virtue of Lemma 1, the coefficient $\sigma$ in $(10.5)-(10.8)$, is equal to 0 . Hence we have

$$
\begin{aligned}
& f_{j}(\lambda)=(\lambda-1)^{3}(\lambda+1) \text { in case } 1, \\
& f_{j}(\lambda)=(\lambda+1)^{3}(\lambda-1) \text { in case } 2, \\
& f_{j}(\lambda)=(\lambda+1)(\lambda-1)\left(\lambda^{2}-\omega_{4} \lambda+1\right) \text { in case } 3, \\
& f_{j}(\lambda)=(\lambda+1)(\lambda-1)\left(\lambda^{2}-\omega_{5} \lambda+1\right) \text { in case } 4 .
\end{aligned}
$$


'Because of $(9.14),(9.15)$, the quadratic polynomials in $\left(10.7^{\prime}\right),\left(10.8^{\prime}\right)$ have only negative real roots. Using formula (7.11), one obtains as results

$$
\begin{aligned}
& \alpha_{1}^{j}=\operatorname{Re} \varrho_{1}{ }^{j}=-\frac{1}{2}, \quad \alpha_{1}^{j}=\operatorname{Re} \varrho_{1}^{j}=0 \quad(l=2,3,4) \quad \text { in case } 1, \\
& \alpha_{1}^{j}=\operatorname{Re} \varrho_{l}^{j}=-\frac{1}{2} \quad(l=1,2,3) ; \quad \alpha_{4}^{j}=\operatorname{Re} \varrho^{j}=0 \quad \text { in the cases } 2-4 .
\end{aligned}
$$

Next, we consider the cases with det $\left(\gamma\left(a_{j}\right)\right)=1$. Particulärly, we have

Case $5: f_{j}(\lambda)=\lambda^{4}+\sigma \hat{i}^{2}+1=0$,

(for nodes $a_{j}$ of the type $(1-8),(1-9),(2-3),(2-5),(3-4),(4-5),(6-8),(6-9)$, $(7-8),(7-9))$,

Case 6: $f_{j}(\lambda)=\lambda^{4}-\omega_{1} \lambda^{3}+\sigma \lambda^{2}-\omega_{1} \lambda+1=0$

(type $(1-6))$,

Case 7: $f_{j}(\lambda)=\lambda^{4}-\omega_{2}^{\prime} \lambda^{3}+\sigma \hat{\lambda}^{2}-\omega_{2} \lambda+1=0$

(type $(1-7))$;

Case 8: $f_{j}(\lambda)=\lambda^{4}-\omega_{2} \lambda^{3}+\sigma \lambda^{2}-\omega_{3} \lambda+1=0$

(type $(2-4)$ and $(3-5))$,

Case 9: $f_{j}(\lambda)=\lambda^{4}-\omega_{6} \lambda^{3}+\sigma \lambda^{2}-\omega_{6} \lambda+1=0$

(type $(\dot{6}-7))$,

Case 10: $f_{j}(\dot{\lambda})=\lambda^{4}+4 \hat{\lambda}^{3}+\sigma \hat{\lambda}^{2}+4 \lambda+1=0$

(type $(8-9))$.

Here, $\sigma$ is a reali number.

In the two last cases, the constant $\sigma$ can be calculated directly. One obtains

$$
f_{j}(\lambda)=\left(\lambda^{2}+2 \frac{1^{\prime}+c_{1}^{2}}{1-c_{1}^{2}} \cdot \lambda+1\right)\left(\lambda^{2}+2 \frac{1+c_{0}^{2}}{1-c_{0}^{2}} \cdot \lambda+1\right)=0 \quad \text { in case } 9
$$

and

$$
f_{j}(\lambda)=(\lambda+1)^{4}=0 \quad \text { in }^{2} \text { case } 10 \text {. }
$$

Bearing in mind $\frac{1+c_{l}^{2}}{1-c_{l}^{2}}>1 \quad(l=0,1)$, we get

$$
\alpha_{l}^{j}=\operatorname{Re} \varrho_{l}^{j}=-\frac{1}{2} \quad(l=1,2,3,4) \quad \text { in the cases } 9 \text { and } 10 .
$$

For the cases 5-8 we first remark that, in consequence of (10.3) and the reality of tho coefficients in (10.13)-(10.16), the following equality holds in the sense of set theory

$$
\left.\left\{\lambda_{1}, \lambda_{2}, \lambda_{3}, \lambda_{4}\right\}=\left\{\bar{\lambda}_{1}, \bar{\lambda}_{2}, \bar{\lambda}_{3}, \bar{\lambda}_{4}\right\}=\lambda_{1}^{-1}, \lambda_{2}{ }^{-1}, \lambda_{3}{ }^{-1}, \lambda_{1}-1\right\}
$$

Lem ma 3: The equations (10.13)-(10.16) have only real roots.

Proof: Let us assume on the contrary that there exists a complex root $\lambda_{1}=p+i q(q \neq 0)$. Then $\lambda_{3}=p-i q$ also solves the characteristic equation $f_{i}(\lambda)=0$.

First, we suppose the existence of another pair of complex roots $\lambda_{3,4}=p_{1} \pm i q_{1}\left(q_{1} \neq 0\right)$. Besides, if $p^{2}+q^{2} \neq 1$, this is a consequence of (10.20). Now, we consider the auxiliary problem $C_{(l-k)}^{*}$ and set $-1<\alpha_{1}^{\prime}=\alpha_{l}{ }^{j}=\arg \left(\lambda_{l}^{j}\right) \leqq 0(l=1, \ldots, 4)$.

The index $\varkappa_{h}$. of the integral equation

$$
\boldsymbol{\Omega}_{p} \mathscr{A} \Phi=A_{1} \boldsymbol{\Omega}_{p} \mathrm{~W}_{1}+A_{2} \boldsymbol{\Omega}_{p} \mathbf{w}_{2}
$$


is obtained by

$$
x_{h^{*}}=\frac{1}{2 \pi}\left[\arg \frac{1}{\prod_{j=1}^{10} \prod_{l=1}^{4}\left(t+z_{0}\right)^{\alpha_{l}^{3}}}\right]_{L}=\frac{1}{2 \pi}\left[\left(t-z_{0}\right)^{20}\right]_{L}=20 .
$$

Consequently, (*) has at least 20 linearly independent solutions. On the other hand, the equation

$$
\mathscr{A} \Phi=A_{1} \mathbf{w}_{1}+A_{2} \dot{w}_{2}+\mathbf{h}, \quad \mathrm{h} \in \operatorname{ker} \Omega_{p}
$$

is equivalent to $(*)$. Because of $(6.11)$, one gets

$$
\operatorname{dim} \text { ker } \boldsymbol{\Omega}_{p} \stackrel{1}{=} 10 \text {. }
$$

For this reason, the equation $(* *)$ (and $(*)$ too) permits at most 14 linearly independent solutions. Therefore the roots $\lambda_{3}, \lambda_{4}$ cannot be complex.

Moreover, it follows that $p^{2}+q^{2}=1$. Let

$$
\begin{aligned}
& \lambda_{1}=p+i q=\mathrm{e}^{2 \pi \alpha i}, \quad \lambda_{2}=p-q i=\mathrm{e}^{-2 \pi \alpha i}\left(-1<\alpha<0 ; \alpha \neq-\frac{1}{2}\right) ; \\
& \lambda_{3}=s, \quad \lambda_{i}=t \quad(s, t \text { real; } s, t \neq 0) .
\end{aligned}
$$

Now, the assumption $s, t<0$ leads to the same contradiction as above.

We show that $s, t>0$ is also impossible. Indeed, the assumption $s, t>0$ implies by $(10.20)$; the relation $t=\frac{1}{s}$. We have.

$$
\lambda_{1}+\lambda_{2}+\lambda_{3}+\lambda_{s}=2 \cos \alpha+\left(s+\frac{1}{s}\right)>0 .
$$

But in regard of $(9.11),(9.12),(9.13)$, the relations

$$
\omega_{l}=\lambda_{1}+\lambda_{2}+\lambda_{3}+\lambda_{4} \leqq 0 \quad\left(l=1,2,3 ; \omega_{l}=0 \text { in case } 5\right)
$$

must hold in contradiction to $(* * *)$.

We still have to consider the case $s<0<t$. Here, (10.20) implies $s=-1, t=1$. Cónsidering. the auxiliary problem $C_{(-k)}^{*}$, one obtains

$$
x_{h^{*}}=\frac{1}{2 \pi}\left[\arg \left(t-z_{0}\right)^{15}\right]_{L}=15 .
$$

Thus, the equation

$$
\Omega_{p} \mathscr{A} \Phi=A_{1} \Omega_{p} w_{1}=A_{2} \Omega_{p} w_{2}
$$

has at least 15 linearly independent solutions. However, the equivalent equation

$$
\mathscr{A} \Phi=A_{1} \mathrm{w}_{1}+A_{2} \mathrm{w}_{2}+\mathrm{h}, \quad \mathrm{h} \in \mathrm{ker} \Omega_{p}:
$$

has at most 14 linearly independent solutions. This is a contradiction, which shows the im. possibility of $s<0<t$.

Consequently, the assumption of existence of a complex root of the characteristic equation . $f_{j}(\lambda)=0$ is not true. Thus Lemma 3 is proved

Now, the values $\alpha_{l}^{j}=\arg . \lambda_{l}^{j}$ of the roots of the characteristic equations $(10.13)-(10.16)$ required for the index calculation can be established. Here the assumptions

$$
\alpha_{1}^{j}=\alpha_{2}^{j}=\alpha_{3}^{j}=\alpha_{4}^{j}=-\frac{1}{2} \text { or } \quad \alpha_{1}^{j}=\alpha_{2}^{j}=\alpha_{3}^{j}=-\frac{1}{2}, \quad \alpha_{1}^{j}=0
$$

'can easily be led to a contradiction by using the auxiliary problem $C_{(l-k)}^{*}$. Further, from the assumption $\alpha_{1}{ }^{i}=\alpha_{2}{ }^{j}=\alpha_{3}{ }^{j}=\alpha_{4}{ }^{j}=0$ follows $\lambda_{1}{ }^{j}+\lambda_{2}{ }^{j}+\lambda_{3}{ }^{j}+\lambda_{4}{ }^{j}>0$, which contradicts to the sign of the coefficient by $\lambda^{3}$ of the characteristic equation. 
The same contradiction occurs in the case

for (10.20) implies for instance

$$
\alpha_{1}^{j}=\alpha_{2}^{j}=\alpha_{3}^{j}=0, \quad \alpha_{4}^{j}=-\frac{1}{2}
$$

$$
\lambda_{1}{ }^{j}=1, \quad \lambda_{1}{ }^{j}=-1 \text { and } \lambda_{1}^{j}+\lambda_{2}{ }^{i}+\lambda_{3}{ }^{j}+\lambda_{1}{ }^{j}=\lambda_{2}{ }^{j}+\lambda_{3}{ }^{j}>0 .
$$

Thus, we have

$$
\alpha_{i}^{j}=\alpha_{2}{ }^{j}=-\frac{1}{2}, \quad \alpha_{3}{ }^{j}=\alpha_{4}{ }^{j}=\frac{1}{2}, \quad \text { in the cases } 5-8 .
$$

Now, we have complete information for determination of the index of the operator $\boldsymbol{\Omega}_{p} \mathscr{A}$.

Using formula (7.11) and (10.11), (10.12), (10.19), (10.21), one obtains for the index $x_{h}$ : in the largest class $h^{*}$

$$
x_{h^{*}}=\frac{1}{2 \pi}\left[\arg \left(t-z_{0}\right)^{s}\right]_{L}=s,
$$

where

$$
\begin{aligned}
s= & s\left(A_{l k}\right) \\
= & \frac{1}{2}\left\{\left(A_{12}+\dot{A}_{13}+A_{14}+A_{15}+A_{27}+A_{29}+A_{36}+A_{38}+A_{46}+A_{49}+A_{57}+A_{58}\right)\right. \\
& +2\left(A_{16}+A_{17}+A_{18}+A_{19}+A_{23}+A_{24}+A_{25}+A_{34}+A_{35}+A_{45}+A_{68}\right. \\
& \left.+A_{69}+A_{78}+A_{29}\right) \\
& +3\left(A_{26}+A_{28}+A_{37}+A_{39}+A_{47}+A_{48}+A_{56}+A_{58}\right) \\
& \left.+4\left(A_{62}+A_{89}\right)\right\} .
\end{aligned}
$$

In comparison with (6.11) one gets

$$
\varkappa_{h^{*}}=s\left(A_{l k}\right)=\dot{r}-q=\operatorname{dim} \operatorname{ker} \Omega_{p} .
$$

For investigation of the operator $\Omega_{p} \mathcal{A}$ in an arbitrary class $h=h\left(a_{j_{1}}, a_{j_{1}}, \ldots, a_{j_{0}}\right)$ let us agree upon the following notation: Let $A_{l k}^{0}$ be the number of the $q$ nodes of type $(l-k)$ in whose neighbourhood the solution $\Phi$ of the equation $(5.10)$ is assumed to belong to the class $H_{\varepsilon}$. Then it is easily seen that

$$
x_{h\left(a_{j_{1}} \cdots, a_{j_{a}}\right)}=x_{h^{*}}-2 s\left(A_{l k}^{0}\right) \text {. }
$$

Especially, we have

$$
x_{h_{\varepsilon}}=-x_{h^{*}}
$$

Thus, the following theorem is proved.

Theorem 1: The system (5.10) is a singular integral equation system of regular type with nonspecial nodes only. Its index $\kappa_{h}$, in the class $h^{*}$ is given by (10.22). Moreover, the relation (10.24) holds.

In an arbitrary class $h=h\left(a_{i_{2}}, \ldots, a_{.}\right)$the index $\varkappa_{h}$ is given by (10.25). 


\section{\$11 Existence of "-regular solutions}

Now one can show that there exists a *-regular solution of the considered contact problem (i.e. a solution of problem $C^{*}$ ). First let us assume that the solution of problem $C^{*}$ is uniquely determined. Then the homogeneous problem $C^{*}$ permits the trivial solution only (the case of nontrivial solutions is treated in $\S 12$ ).

By-the single layer potential setup (5.1) for the solution $\mathbf{u}$ of $C^{*}$ with density vector $\boldsymbol{\Phi}$ satisfying the additional condition

$$
\int_{L} \varphi^{0}(\mathbf{y}) d s_{\mathbf{y}}=0
$$

one obtains the integral equation system

$$
\mathcal{A} \Phi=A_{1} \mathrm{w}_{1}+A_{2} \mathbf{w}_{2}^{\prime}+\mathrm{w}
$$

with the four-component density vector $\Phi$ and the real constants $A_{1}, A_{2}$ to be determined. Using the linear operator $\boldsymbol{\Omega}_{p}$ defined in $\$ 5$, one gets the integral equation
system

$$
\boldsymbol{\Omega}_{p} \mathcal{A} \Phi=A_{1} \boldsymbol{\Omega}_{p} \mathrm{w}_{1}+A_{2} \boldsymbol{\Omega}_{p} \mathrm{w}_{2}+\boldsymbol{\Omega}_{p} \mathbf{W},
$$

which is equivalent to

$$
\mathcal{L} \Phi=A_{1} \mathbf{w}_{1}+A_{2} \mathbf{w}_{2}+\mathbf{w}+\mathbf{h}, \quad \mathrm{h} \in \operatorname{ker} \boldsymbol{\Omega}_{p}
$$

In order to obtain a *-regular solution of $C^{*}$, the systems (5.4), (5.10), (11.1) are considered in the class $h^{*}$. In accordance with Theorem 10.1 , the system $(5.10)$ is of regular type and has nonspecial nodes only. Therefore, the class $h^{*}$ coincides with the
class $H^{*}$.

Let us consider the following linear manifolds:

$$
\begin{aligned}
& \mathfrak{M}=\left\{\Phi \in H^{*} / \Omega_{p} \mathscr{A} \Phi=A_{1} \Omega_{p} \mathbf{w}_{1}+A_{2} \Omega_{p} \mathbf{w}_{2}\right\} \\
& \mathfrak{U}_{0}=\left\{\Phi \in \mathfrak{M} / \int_{L} \varphi^{0}(\mathbf{y}) d s_{\mathbf{y}}=0\right\}
\end{aligned}
$$

Obviously, $\mathfrak{M}$ can also be characterized by

$$
\dot{\mathfrak{M}}=\left\{\boldsymbol{\Phi} \in H^{*} / \mathcal{A} \Phi=A_{1} \mathbf{w}_{1}+A_{2} \mathbf{w}_{2}+\mathrm{h} ; \mathrm{h} \in \operatorname{ker} \boldsymbol{\Omega}_{p}\right\} \text {. }
$$

For the further considerations, the following lemma is crucial.

Lem ma 1: Let the homogeneous problem $C^{*}$ have only the trivial solutions. Then

$$
\begin{aligned}
& \operatorname{dim} \mathfrak{M}=\dot{x}_{n^{\bullet}}+2, \\
& \operatorname{dim} \mathfrak{M}_{0}=\dot{x}_{h^{\bullet}}
\end{aligned}
$$

The proof makes use of Theorems 10.1 and 7.1. Because of $(6.3)$ and the definition of $\mathbf{w}_{1}, \mathbf{w}_{2}$ (see $\left.\S 5\right)$; we have $\mathfrak{L}\left\{\mathbf{w}_{1}, \mathbf{w}_{2}\right\} \cap \operatorname{ker} \boldsymbol{\Omega}_{p}=\mathbf{0}$. For this reason, a $\left(\varkappa_{h^{*}}+2\right)$-dimensional linear manifold is situated on the right-hand side of the equation

$$
\mathscr{A} \dot{\boldsymbol{\Phi}}=A_{1} \mathbf{w}_{1}+A_{2} \mathbf{w}_{2}+\mathbf{h}, \quad \mathbf{h} \in \operatorname{ker} \boldsymbol{\Omega}_{p}
$$

defining $\mathfrak{M}$. Now Lemma 10.1 implies

$$
\operatorname{dim} \mathfrak{M} \leqq x_{h^{*}}+2 \text { and } \operatorname{dim} \mathfrak{M}_{0} \leqq x_{A^{*}} .
$$


Let us consider the linear subspace

$$
\operatorname{ker}\left(\boldsymbol{\Omega}_{p} \mathcal{A}\right)=\left\{\Phi \in H^{*} / \boldsymbol{\Omega}_{p} \mathcal{A} \boldsymbol{\Phi}=\mathbf{0}\right\}
$$

By the aid of Noether's theorems, we have

$$
x_{h^{*}} \leqq \operatorname{ker}\left(\Omega_{p} \mathcal{A}\right) \leqq x_{h^{*}}+2 .
$$

Let us define

$$
q=\operatorname{dim} \operatorname{ker}\left(\boldsymbol{\Omega}_{p} \mathcal{A}\right)-x_{h^{*}} .,
$$

Then the inequalities $0 \leqq q \leqq 2$ hold. In the case where $q=2$, the proposition (11.5a) follows immediately. The assumption $0 \leqq q \leqq 1$ implies that the homogeneous adjoint integral equation system

$$
\left(\boldsymbol{\Omega}_{\boldsymbol{p}} \mathcal{A}\right)^{\prime} \Psi=\mathbf{0}
$$

has exactly $q$ linearly independent solutions. By a simple consequence of Noether's , theorems one can easily see that there exist $(2-q)$ linearly independent vectors $\dot{\mathbf{m}}_{1}, \ldots, \mathbf{m}_{2-q} \in \mathfrak{L}\left\{\mathbf{w}_{1}, \mathbf{w}_{2}\right\}$ such that the equations

$$
\boldsymbol{\Omega}_{p} \mathcal{A} \boldsymbol{\Phi}=\mathbf{m}_{\mathbf{i}} \quad(i=1, \ldots, 2-q)
$$

are solvable. Assume that $\Phi_{1}, \ldots, \Phi_{2-q}$ are any solutions of the $(2-q)$ equations (**). Moreover, let the vectors $\boldsymbol{\Phi}_{2-q+1}, \ldots, \boldsymbol{\Phi}_{\varkappa_{h^{*}}}+2$ be linearly independent and form a basis of the linear space $\operatorname{ker}\left(\boldsymbol{\Omega}_{p} \mathcal{A}\right)$. Then the vectors $\boldsymbol{\Phi}_{1}, \boldsymbol{\Phi}_{2}, \ldots, \boldsymbol{\Phi}_{\varkappa_{x_{h^{*}+2}}}$ are linearly independent and belong to $\mathfrak{R}$. Taking into account $(*)$, the proposition $(11.5 \mathrm{a})$ is proved.

By a simple consideration, one can select $\varkappa_{h}$. linearly independent vectors from $\mathfrak{L}\left\{\boldsymbol{\Phi}_{1}, \boldsymbol{\Phi}_{2}, \ldots, \boldsymbol{\Phi}_{x_{h^{*}+2}}\right\}$ which satisfy the additional condition $(5.2)$. Thus, using $(*)$, $(11.5 \mathrm{~b})$ is also proved

Let $\Psi_{1}, \ldots, \Psi_{q}$ be linearly independent solutions of (11.8). Then we have by Noether's theorems the necessary and sufficient conditions

$$
\int_{L} \Psi_{\mathbf{i}}(\mathbf{y}) \cdot \mathbf{v}(\mathbf{y}) d s_{\mathbf{y}}=\mathbf{0} \quad(i=1, \ldots, q) \quad \text { ' }
$$

for solvability of $\Omega_{p} \mathscr{A} \Phi=\mathbf{v}\left(\Psi_{i} \cdot \mathbf{v}\right.$ is the scalar product in $\left.\mathbf{R}^{\mathbf{4}}\right)$.'

Corollary 1: We have

$$
\operatorname{rank}\left[\begin{array}{cc}
\int_{L} \Psi_{1} \Omega_{p} w_{1} d s \ldots \int_{L} \Psi_{q} \Omega_{p} \mathbf{w}_{1} d s \\
\int_{L} \Psi_{1} \Omega_{p} \mathbf{w}_{2} d s \ldots \int_{L} \Psi_{q} \Omega_{p} \mathbf{w}_{2} d s
\end{array}\right]=q_{:}
$$

Indeed, the rank cannot be greater than $q$. Suppose that the rank were less then $q$. Then we could find at least $q+1$ linearly independent vectors $\mathbf{m}_{1}, \ldots, \mathbf{m}_{q+1} \in \mathfrak{L}\left\{\mathbf{w}_{1}, \mathbf{w}_{2}\right\}$ such that the equations $(* *)^{\prime}$ are solvable for the vectors $\mathbf{m}_{i}(i=1, \ldots, q+1)$. Clearly, this consequence would contradict (11.5a). Thus the corollary is proved

Corollary 2 : Let $\boldsymbol{\Phi}_{1}, \boldsymbol{\Phi}_{2}, \ldots, \boldsymbol{\Phi}_{x_{h^{*+2}}}$ be a basis of the linear manifold 1$)$. Without loss of generality one can assume

$$
\boldsymbol{\Phi}_{1}, \ldots, \boldsymbol{\Phi}_{x_{h^{*}}} \in \mathfrak{M}_{0} ; \quad \boldsymbol{\Phi}_{x_{h^{*}+1}}, \boldsymbol{\Phi}_{x_{h^{*}+2}} \notin \mathfrak{M}_{0}
$$

Then

$$
\operatorname{rank}\left[\int_{L} \boldsymbol{\varphi}_{x_{h^{*}+1}}^{0}(\mathbf{y}) d s_{\mathrm{y}}, \quad \int_{L} \boldsymbol{\varphi}_{x_{h^{*}+2}}^{0}(\mathbf{y}) d s_{\mathbf{y}}\right]=\mathbf{2}
$$


Of course, the rank cannot be greater than 2 . But the assumption $<2$ would imply that $\operatorname{dim} \mathfrak{M}_{0} \geqq x_{h^{*}}+1$, in contradiction to (11.5b). Thus, (11.10) is shown

Now we consider the problem $C^{*}$ with contact data $\mathbf{w}_{0}$ contained in the linear space

$$
\begin{aligned}
& \mathfrak{P}=\left\{\mathrm{w}_{0} / \mathrm{w}_{0}=A_{1} \mathrm{w}_{1}+A_{2} \mathbf{w}_{2}+\mathbf{h} ; \quad \mathrm{h} \in \operatorname{ker} \boldsymbol{\Omega}_{p},\right. \\
& \left.A_{1}, A_{2} \text { arbitrary real constants }\right\}
\end{aligned}
$$

We have $\operatorname{dim} \mathfrak{\Re}=x_{h}$ * +2 .

Le mima 2:- Let the homogeneous problem $C^{*}$ have only the trivial solution. Then the problem $C^{*}$ with contact data $\mathbf{w}_{0} \in \Re$ has exactly one solution $\mathbf{u}$. This solution $\mathbf{u}$ can be represented by

$$
\begin{aligned}
& \mathbf{u}^{0}(\mathbf{x})=A_{1} \mathbf{c}^{1}+A_{2} \mathbf{c}^{2}+\nabla^{0}\left(\mathbf{x} ; \varphi^{0}\right) \quad \text { for } \quad \mathbf{x} \in D_{0} \\
& \mathbf{u}^{\mathbf{1}}(\mathbf{x})=\nabla^{1}\left(\mathbf{x} ; \varphi^{1}\right) \text { for } \quad \mathbf{x} \in \dot{D}_{1},
\end{aligned}
$$

where the vector $\Phi=\left[\begin{array}{l}\dot{\varphi}^{0} \\ \varphi^{1}\end{array}\right]$ and the constants $A_{1}, A_{2}$ solve the integral equation

$$
\boldsymbol{\Omega}_{p} \mathcal{A} \boldsymbol{\Phi}=\dot{A}_{1} \boldsymbol{\Omega}_{p} \mathbf{w}_{1}+A_{2} \boldsymbol{\Omega}_{p} \mathbf{w}_{2}
$$

and the additional condition (5.2).

Proof: In virtue of (11.5a), the equation (11.15) has exactly $x_{h^{*}}+2$ linearly independent solutions $\boldsymbol{\Phi}_{1}, \ldots, \boldsymbol{\Phi}_{x_{h^{\bullet}+2}}$. Assume that the conditions of the corollary are satisfied. Then, using Theorem 4.2 , one finds that the potentials

$$
\mathbf{V}\left(\mathbf{x} ; \boldsymbol{\Phi}_{k}\right)=\left[\begin{array}{c}
\mathbf{V}^{0}\left(\mathbf{x} ; \boldsymbol{\varphi}^{0}\right) \\
\mathbf{V}^{1}\left(\mathbf{x} ; \boldsymbol{\varphi}^{1}\right)
\end{array}\right], \quad\left(k=1, \ldots, x_{h^{*}}\right)
$$

are *-regular solutions of $C^{*}$ with contact data belonging to $\mathfrak{B}$. By nondifficult considerations (using the equivalence property of $V^{1}\left(x ; \varphi^{1}\right)$ and the results on integral equation (4.15)), one obtains the linear independence of the potentials (11.16). Consider the vectors

$$
V_{i}=\left\{\begin{array}{lll}
c^{i} & \text { for } & x \in D_{0} \\
0 & \text { for } & x \in D_{1}
\end{array} ;(i=1,2)\right.
$$

These vectors $\dot{V}_{i}$ have the contact data $w_{1}, w_{2}$ respectively. Because of (4.7) the $\varkappa_{h^{*}}$ +2 vectors

$$
\mathbf{V}\left(\mathbf{x} ; \boldsymbol{\Phi}_{1}\right), \ldots, \mathbf{V}\left(\mathbf{x} ; \boldsymbol{\Phi}_{x h^{*}}\right), \quad \mathbf{V}_{1}, \dot{\mathbf{V}}_{2}
$$

are linearly independent. Thus, the vectors represent a basis in the linear space of solutions of $C^{*}$ with contact data in $\mathfrak{\Re}$. The lemma is proved

Now, we obtain

Theorem 1: Let $L \in C^{2, \beta}(0<\beta \leqq 1)$. Let the homogeneous problem ${ }^{*} *$ have only the trivial solution. Let the contact data $\mathbf{f}, f, \mathbf{f}_{k}, f_{k}, l_{k}, g, g, g_{k}, g_{k}, h_{k}$ satisfy the assumptions of $\S 2$ and the compatibility conditions $(1.6 \mathrm{a})-(1.6 \mathrm{x})$. Then there exists $a^{*}$-regular solution $\mathbf{u}$ of the problem $C^{*}$. $\mathbf{u}$ is representable in the form (11.14) (see also (5.1)). $\Phi$ and the constants $A_{1}, A_{2}$ are uniquely determined by the iniegral equation (5.10) with the additional condition (5.2). 
- For proof consider the system (5.10) with arbitrary $A_{1}, A_{2}$. First we show that (5.10) has a solution $\tilde{\Phi}$ for suitable constants $A_{1}{ }^{0}, A_{2}{ }^{0}$. In the case $q=0$ (see (11.7)) this is immediately seen. Otherwise, the formula (11.11) guarantees the existence if a suitable pair $A_{1}{ }^{0}, A_{2}{ }^{0}$ and a vector $\dot{\Phi}$ with

$$
\dot{\boldsymbol{\Omega}}_{p} \mathcal{A} \tilde{\boldsymbol{\Phi}}=A_{1}{ }^{0} \boldsymbol{\Omega}_{p} \mathrm{w}_{1}+A_{2}{ }^{0} \boldsymbol{\Omega}_{p} \mathrm{w}_{2}+\boldsymbol{\Omega}_{p} \mathrm{w} .
$$

At the same time, we have

$$
\mathcal{A} \dot{\boldsymbol{\Phi}}=A_{1}{ }^{0} \mathbf{w}_{1}+A_{2}{ }^{0} \mathbf{w}_{2}+\overline{\mathbf{h}} ; \quad \tilde{\mathbf{h}} \in \operatorname{ker} \boldsymbol{\Omega}_{\dot{p}}
$$

The general solution $\hat{\boldsymbol{\Phi}}$ of $(11.18)$ is

$$
\hat{\boldsymbol{\Phi}}=\tilde{\boldsymbol{\Phi}}+\sum_{i=1}^{x_{h}^{*+2}} C_{i} \bar{\Phi}_{i}, \quad C_{i}-\text { arbitrary constants, }
$$

with the vectors $\dot{\Phi}_{i}$ of Corollary 2 . Using this corollary, one can assume that $\overline{\boldsymbol{\Phi}}$ fulfils the condition (5.2). Finally, because of Lemma 2 there exists a vector $\overline{\boldsymbol{\Phi}}$ satisfying (5.2) such that the vectors

$$
\mathbf{u}^{0}(\mathbf{x})=-A_{1}{ }^{0} \nabla_{1}-A_{2}{ }^{0} \mathbf{V}_{2}+\nabla^{0}\left(\mathbf{x} ; \bar{\varphi}^{0}\right), \quad \mathbf{u}^{1}(\mathbf{x})=\mathbf{V}^{1}\left(\mathbf{x} ; \bar{\varphi}^{1}\right)
$$

are solutions of $C^{*}$ with contact data $-\dot{A}_{1}{ }^{0} \mathrm{w}_{1}-A_{2}{ }^{0} \mathrm{w}^{2}-\tilde{\mathrm{h}}$. Obviously, by setting

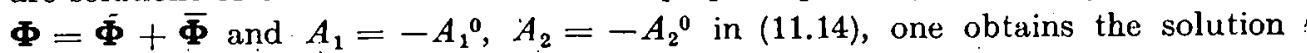
of problem $C^{*}$.

The uniqueness of $\boldsymbol{\Phi}_{1}$ and $A_{1}, A_{2}$ is a consequence of the equivalence of $\nabla^{1}$ and (4.15)

\section{$\$ 12$ *-regular solutions in the caso of eigensolutions}

In the case of nontrivial solutions of the homogeneous problem $C^{*}$ some more sophisticated considerations are necessary. The general result is here that a solution of the inhomogeneous problem $C^{*}$ exists if and only if the contact data satisfy the physical conditions for solvability of $\S 3$. Moreover, the solution u of $C^{*}$ can always be represented in the form (5.1), provided that the physical conditions are fulfilled. The aim of the present section is to demonstrate the general ideas of the proof. Especially, the two examples of $\S 3$ with exactly one eigenvalues are considered in detail. For the remaining examples of $\S 3$ the results are given in outline.

First, let us assume that the homogeneous problem $C^{*}$ permits $h$ linearly indepen-: dent solutions $(h \geqq 1)$. Furthermore, let us suppose that $h$ linearly independent . solutions $\Psi_{1}, \Psi_{2}, \ldots ., \Psi_{h}$ of the homogeneous adjoint system

$$
\left(\boldsymbol{\Omega}_{p} \mathcal{A}\right)^{\prime} \Psi_{\mathbf{i}}=\mathbf{0} \quad(i=1, \ldots, h)
$$

are explicitly known. These vectors $\Psi_{1}, \ldots, \Psi_{h}$ imply the solvability conditions

$$
\int_{L} \Psi_{i} \cdot\left(A_{1} \boldsymbol{\Omega}_{p} \mathbf{w}_{1}+A_{2} \boldsymbol{\Omega}_{p} \mathbf{w}_{2}+\boldsymbol{\Omega}_{p} \mathbf{w}\right) d s=0 \quad(i=1,2, \ldots, h)
$$

for the system (5.10). By the second assumption

$$
\int_{L} \Psi_{i} \Omega_{p} \mathbf{w}_{j} d s=0 \quad(i=1, \ldots, h ; j=1,2),
$$

the solvability conditions reduce to

$$
\int_{L} \dot{\Psi}_{i} \Omega_{p} \mathbf{w} d s=0 \quad(i \doteq 1 ; 2, \ldots, h) .
$$


With the accepted assumptions, the general pattern for solving the problem $C^{*}$ is the following. As in $\S 11$ we consider the sets $\mathfrak{M}, \mathfrak{M}_{0}$ ((11.2), (11.3)). The crucial lemma is

Lem ma 1: Let the above-mentioned assumptions be satisfied. Further, let the homogeneous problem $C^{*}$ have exactly $h$ linearly independent solutions. Then

where

$$
\begin{aligned}
& \operatorname{dim} \mathfrak{M}=h+d+\varkappa_{h^{*}}, \\
& \operatorname{dim} \mathfrak{U K}_{0}=h+d+\varkappa_{h^{*}}-2,
\end{aligned}
$$

$$
d=\operatorname{dim} \mathfrak{L}\left\{\mathbf{w}_{1}, \mathbf{w}_{2}\right\} \text {. }
$$

For proof, the argumentation of Isemma 11.1 is essentially repeated. In distinction from (11.6), one gets

$$
h+\mathscr{x}_{h^{*}} \leqq \operatorname{dim} \operatorname{ker} \Omega_{p^{\prime}} \leqq h+d+\varkappa_{h^{*}}
$$

in consequence of Noether's theorems and of the assumptions concerning the system [12.1)

In virtue of (12.5), the system (12.1) has $h+q$ linearly independent solutions $\Psi_{1}, \Psi_{2}, \ldots, \Psi_{n+q}$. Here, $q$ is defined by

$$
q=\operatorname{dim} \operatorname{ker} \boldsymbol{\Omega}_{p^{A}} \mathcal{A}-\varkappa_{h^{\circ}}-h .
$$

Let $\Psi_{1}, \Psi_{2}, \ldots, \Psi_{h}$ be the above-mentioned solutions of $(12.1)$, but $\Psi_{h+1}, \ldots, \Psi_{h+Q}$ additional solutions. Similarly as in $\S 11$, we obtain the following corollaries.

Corollary 1: We have

$$
\operatorname{rank}\left[\begin{array}{c}
\int_{L} \Psi_{h+1} \Omega_{p} \mathbf{W}_{1} d s \ldots \int_{L} \Psi_{h+q} \Omega_{p} \mathbf{W}_{1} d s \\
\int_{L} \Psi_{h+1} \Omega_{p} \mathbf{W}_{2} d s \ldots \int_{L} \Psi_{h+q} \boldsymbol{\Omega}_{p} \mathbf{W}_{2} d s
\end{array}\right]=q
$$

Corollary 2: Let $\boldsymbol{\Phi}_{1}, \ldots, \boldsymbol{\Phi}_{h+d+x_{h}}$ be a basis of the linear space $\mathfrak{M}$. Then we can assume

$$
\boldsymbol{\Phi}_{1}, \ldots, \boldsymbol{\Phi}_{h+d-2+x_{h^{*}}} \in \mathfrak{M}_{0} ; \quad \boldsymbol{\Phi}_{h+d-1+x_{h^{*}}}, \quad \boldsymbol{\Phi}_{h+d+x_{h^{*}}} \notin \mathfrak{M}_{0} .
$$

Further, we have

$$
\operatorname{rank}\left[\int_{L} \varphi_{h+d-1+x_{h^{*}}}^{0} d s, \int_{L} \varphi_{h+d+x^{*}}^{0} d s\right]=2
$$

By insignificant modification of the considerations in $\S 11$, the following theorem can be proved.

'Theorem-1: Let the above-mentioned assumptions be satisfied. Moreover, let $L \in C^{2, \beta}$ $(0<\beta \leqq 1)$ and let the homogeneous problem $C^{*}$ have exactly $h$ linearly independent solutions. Let the contact data $\mathbf{f}, f, \mathbf{f}_{k}, f_{k}, l_{k}, g, g, g_{k}, g_{k}, h_{k}$ satisfy the assumptions of $\S 2$, besides these the compatibility conditions (1.6a)-(1.6x) and, additionally, the physical solvability conditions of $\S 3$. Then there exists $a^{*}$-regular solution $\mathrm{u}$ of the problem $C^{*}$. $\mathbf{u}$ is representable in the form (11.14). $\boldsymbol{\Phi}$ and the constants $A_{1}, A_{2}$ are determined by the integral equation (5.10) with the additional condition (5.2). Furthermore, the physical conditions coincide, of necessity, with the relations (12.3).

For instance, let us consider the second example of $\S 3$ with one eigensolution $\left(L=L_{2} \cup L_{5} \cup L_{7}\right.$, see [2: Figure 2]). Here, the homogeneous adjoint integral equation system is

$$
\mathbf{A}^{\top}(\mathbf{z}) \Psi(z)+\frac{1}{\pi} \int_{L}\left[\mathbf{K}^{\top}(\mathbf{y}-z)+p \mathbf{R}^{\top}(\mathbf{y}-\mathbf{z})\right] \Psi(\mathbf{y}) d s_{\mathbf{y}}=\mathbf{0}
$$


with

$$
\mathbf{A}^{\top}(\mathbf{z})=\left\{\begin{array}{rrrr}
0 & n_{2} & 0 & n_{1} \\
0 & -n_{1} & 0 & n_{2} \\
-n_{2} & 0 & 0 & n_{1} \\
n_{1} & 0 & 0 & n_{2}
\end{array}\right] \text { for } \mathrm{z} \in L_{2},
$$

and $\mathbf{K}^{\top}(\mathbf{y}-\mathbf{z})=$

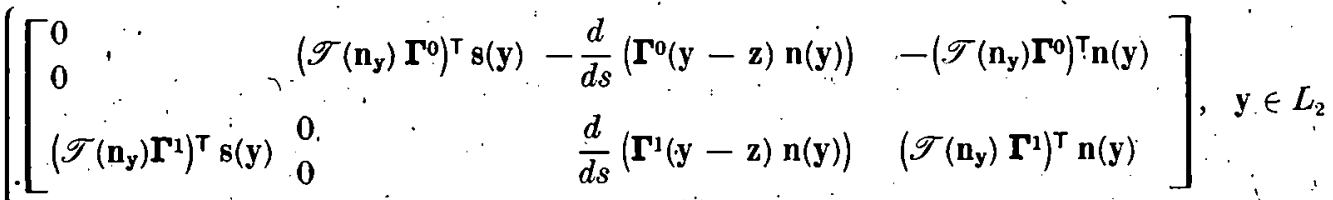

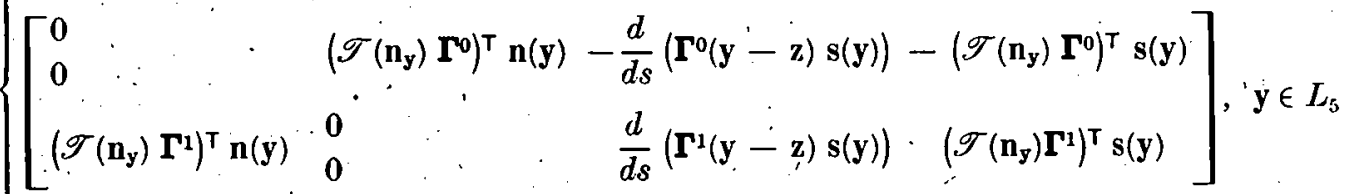

$$
\begin{aligned}
& {\left[\begin{array}{cccc}
\left(\mathscr{T}\left(\mathbf{n}_{\mathbf{y}}\right) \boldsymbol{\Gamma}^{\mathbf{0}}(\mathbf{z}-\mathbf{y})\right)^{\top}-0 & 0 \\
\mathbf{0} & 0 & 0 \\
0 & 0 & \left(\mathscr{T}\left(\mathbf{n}_{\mathbf{y}}\right) \boldsymbol{\Gamma}^{\mathbf{1}}(\mathbf{y}-\mathbf{z})\right)^{\top}
\end{array}\right], \quad \mathbf{y} \in L_{\mathbf{\gamma}}}
\end{aligned}
$$

Because of the special geometrical shape of $L$, we have $\dot{\mathbf{n}} \cdot \mathbf{c}_{v}{ }^{3}=0, \mathbf{s}=B \mathbf{c}_{v}{ }^{3}$ on $L_{2} ; B$ is a function that is constant on every arc $S_{i}$ belonging to $L_{2}$. Analogously, we have $\mathbf{s} \cdot \mathbf{c}_{v}{ }^{3}=0, \mathbf{n}=D(\mathbf{z}) \mathbf{c}_{v}{ }^{3}$ for $\mathbf{z} \in L_{5}$. We define

$$
\Psi(z)=\left\{\begin{array}{c}
{\left[\begin{array}{c}
\mathbf{s} \cdot \mathbf{c}_{v}{ }^{3} \\
0 \\
0 \\
0
\end{array}\right] \text { for } \quad \mathrm{z} \in L_{2}} \\
{\left[\begin{array}{c}
\mathbf{n} \cdot \mathbf{c}_{v}{ }^{3} \\
0 \\
0 \\
0
\end{array}\right] \text { for } \quad z \in L_{5}} \\
{\left[\begin{array}{c}
0 \\
0 \\
\mathbf{c}_{v}^{3}
\end{array}\right] \text { for } \quad z \in L_{i}}
\end{array}\right.
$$


Then the equation (12.1) is equivalent to the identity

$$
\mathbf{c}_{v}{ }^{3}(\mathbf{z})+\frac{1}{\pi} \int_{L}\left(\mathscr{T}\left(\mathbf{n}_{\mathbf{y}}\right) \Gamma^{1}(\mathbf{y}-\mathbf{z})\right)^{\top} \mathbf{c}_{v}{ }^{3}(\mathbf{y}) d s_{\mathbf{y}}=\mathbf{0},
$$

which follows from (4.4). The vector (12.11) gives rise to the necessary condition for solvability (3.8) stated in $\$ 3$. Our considerations imply the sufficiency of these physical conditions in the class of *-regular vectors.

The considerations for the first example with one eigensolution of $\S 3$ ( $L=L_{1} \cup L_{4}$ $\cup L_{7}$, see [2: Figure 1]) are a little different from the above ones. First; one obtains the following lemma.

Le mima 2: Let $\Phi$ be an arbitrary solution of the integral equation

$$
\boldsymbol{\Omega}_{p} \mathcal{A} \boldsymbol{\Phi}=A_{1} \boldsymbol{\Omega}_{p} \mathbf{w}_{1}+A_{2} \boldsymbol{\Omega}_{p} \mathbf{w}_{2}
$$

in the considered special case of the problem $C^{*}$. Then the relation

$$
\int_{L}\left(\mathbf{c}_{\omega} \cdot \varphi^{0}\right) d s=\mathbf{0}
$$

holds true.

For proof, the equation (12.12) (concerning the explicit form of the operator $\Omega_{p} d$ see $(5.10)-(5.14))$ is multiplied in the sense of scalar product with the following factors:

$\left[\begin{array}{l}0 \\ 0 \\ \mathrm{c}_{\omega}\end{array}\right]$ for $\mathrm{z} \in L_{1},\left[\begin{array}{l}0 \\ 0 \\ 0 \\ \left(\mathrm{~s} \cdot \mathrm{c}_{\omega}\right)\end{array}\right]$ for $\mathrm{z} \in L_{4}$ and $\left[\begin{array}{c}-\mathbf{c}_{\omega} \\ \mathrm{c}_{\omega}\end{array}\right]$ for $\mathrm{z} \in L_{3}$.

It is not difficult to see that the scalar product on the right-hand side is 0 . Using the formulas (5.12) - (5.14), the left-hand side can be calculated. Taking into account the relations $\mathbf{s}= \pm \mathbf{c}_{\omega}$ for $\mathrm{z} \in L_{4}$, one obtains the formula

$$
\begin{aligned}
& \int_{L} c_{\omega} \cdot\left\{\varphi^{0}(z)+\varphi^{1}(z)+\frac{1}{\pi} \cdot \int_{L}\left(\mathscr{T}\left(n_{y}\right) \dot{\Gamma}^{1}(y-z)\right)^{\top} \varphi^{1}(y) d s_{y}\right. \\
& \left.-\frac{1}{\pi} \int_{L}\left(\mathscr{T}\left(\mathbf{n}_{\mathbf{y}}\right) \boldsymbol{\Gamma}^{0}(\mathbf{y})\right)^{\top} \varphi^{0}(\mathbf{y}) d s_{y}\right\} d s_{\mathbf{z}}=\mathbf{0} .
\end{aligned}
$$

The integrating order of the double integral can be altered. Using formula (4.4), we get $2 \int_{L} \mathbf{c}_{\omega} \cdot \boldsymbol{\varphi}^{0}(\mathbf{z}) d s_{\mathbf{z}}=0$. The lemma is proved

Applying the same procedure to the equation

$$
\boldsymbol{\Omega}_{p} \mathcal{A} \Phi=\Omega_{p} \mathbf{w}+A_{1} \Omega_{p} \mathbf{w}_{1}+A_{2} \Omega_{p} \mathbf{w}_{2},
$$

one obtains

$$
2 \int_{L} \mathbf{c}_{\omega} \cdot \boldsymbol{\varphi}^{0} d s=\int_{L_{1}} \mathbf{c}_{\omega} \cdot g d s+\int_{L_{s}}\left(\mathbf{s} \cdot \mathbf{c}_{\omega}\right) g d s+\int_{L,} \mathbf{c}_{\omega} \cdot\left(\mathrm{g}_{1}-\mathrm{g}_{0}\right) d s .
$$

This formula shows that the relation (12.13) is fulfilled if and only if the physical condition (3.7) is satisfied. In order to study the problem $C^{*}$, let us consider the 
equation

$$
\boldsymbol{\Omega}_{p} \mathcal{A} \boldsymbol{\Phi}=A_{1} \boldsymbol{\Omega}_{p} \mathbf{w}_{1}+A_{2} \boldsymbol{\Omega}_{p} \mathbf{w}_{2},
$$

which is equivalent to

$$
\mathcal{A} \Phi=A_{1}^{\prime} \mathbf{w}_{1}+A_{2} \mathbf{w}_{2}+\mathrm{h}, . \quad \mathrm{h} \in \operatorname{ker} \boldsymbol{\Omega}_{p} .
$$

We define the linear manifolds

and

$$
\mathfrak{M}=\left\{\boldsymbol{\Phi} \in H^{*} / \boldsymbol{\Omega}_{p} \mathcal{A} \boldsymbol{\Phi}=A_{1} \boldsymbol{\Omega}_{p} \mathbf{w}_{1}+A_{2} \boldsymbol{\Omega}_{p} \mathbf{w}_{2}\right\}
$$

$$
\mathfrak{M}_{0}=\left\{\Phi \in \mathfrak{M} / \int_{L} \boldsymbol{\varphi}^{0} d s=0\right\}
$$

By nondifficult considerations (especially, using [5: Hilfssatz 9.1] and the continuity of the potential of single layer), formula (12.13) implies that the equation

$$
\boldsymbol{\Omega}_{p} \mathscr{A} \Phi=A_{1} \boldsymbol{\Omega}_{p} \mathrm{w}_{1}+A_{2} \boldsymbol{\Omega}_{p} \mathrm{w}_{2}
$$

is unsolvable for at least one pair $\tilde{A}_{1}, \tilde{A}_{.2}$. Let $\tilde{\mathrm{w}}=\tilde{A}_{1} \mathrm{w}_{1}+\tilde{A}_{2} \mathrm{w}_{2}$. Then the equation

$$
\Omega_{p} \mathcal{A} \Phi=\tilde{A} \Omega_{p} \overline{\mathbf{w}}, \quad \tilde{A} \neq 0
$$

is unsolvable.

Lem ma 3: In the considered case we have

$$
\operatorname{dim} \mathfrak{M}=x_{h^{*}}+2 \text { and } \operatorname{dim} \mathfrak{M}_{0}=x_{h^{*}}+1 .
$$

Proof: Bearing in mind (12.16), the relation $\operatorname{dim}$ ker $\Omega_{p}=\varkappa_{h^{*}}$ and the unsolvability of equation (12.18), one obtains

$$
\operatorname{dim} \mathfrak{M} \leqq x_{h^{*}}+2 \text { and } \operatorname{dim} \mathfrak{M}_{0} \leqq x_{h^{*}}+1
$$

In order to prove $\operatorname{dim} \mathfrak{M}=x_{h^{*}}+2$, we mention the inequalities

$$
\varkappa_{h^{*}}+1 \leqq \operatorname{dim} \operatorname{ker}\left(\Omega_{p} \mathscr{A}\right) \leqq x_{h^{*}}+2 \text {. }
$$

By Noether's theorems, the first inequality is a consequence of the unsolvability of equation (12.18). In the case where $\operatorname{dim} \operatorname{ker}\left(\Omega_{p} \mathcal{A}\right)=x_{h^{*}}+2$ the equation $\operatorname{dim} \mathfrak{M}$ $=x_{n^{*}}+2$ is evident. Assume $\operatorname{dim} \operatorname{ker}\left(\Omega_{p} \mathscr{A}\right)=x_{n^{*}}+1$. Then the adjoint homogeneous equation $\left(\boldsymbol{\Omega}_{p} \mathcal{A}\right)^{\prime} \Psi=0$ has exactly one linearly independent solution. Now, using the unsolvability of $(12,18)$, one can easily prove that the linear space $\mathfrak{L}\left\{\mathbf{w}_{1}, \mathbf{w}_{2}\right\}$ contains a nontrivial vector $\hat{w}$ such that

$$
\boldsymbol{\Omega}_{p} \mathscr{A} \boldsymbol{\Phi}=\boldsymbol{\Omega}_{\boldsymbol{p}} \hat{\mathrm{w}}
$$

is solvable. Thus, $\operatorname{dim} \mathfrak{M}=\varkappa_{h^{*}}+2$ is also true.

- Let $\Phi_{1}, \ldots, \Phi_{x^{*}+2}$ be a basis of $\mathfrak{M}$. Taking into account (12.13), we can assume without loss of generality $\int_{L} \varphi_{i}{ }^{0} d s=0$ for $i=1, \ldots, \varkappa_{h^{*}}+1$. 'This implies dim $\mathfrak{M H}_{0}$ ' $\geqq \varkappa_{h^{*}}+1$. Thus, $\operatorname{dim} \mathfrak{M}_{0}=\varkappa_{h^{*}}+1$ is also proved

Corollary 3: Let $\boldsymbol{\Phi}_{1}, \ldots, \Phi_{x_{h}+2}$ be a basis of $\mathfrak{M}$, chosen as in the proof of Lemma 3. Then we have

$$
\int_{L} \boldsymbol{\varphi}_{x_{h}+2}^{0} \cdot \mathbf{c}_{\omega}{ }^{1} d s \neq \dot{0}
$$

The corollary is proved in analogy to Corollary 2 of $\$ 11$ 
Obviously, the adjoint equation $\left(\Omega_{p} \mathcal{A}\right)^{\prime} \Psi=0$ permits either 1 or 2 linearly independent solutions. Let $\Psi_{1}, \ldots, \Psi_{k}(k=1,2)$ be the solutions of the adjoint equation. Then the following corollary holds.

Corollary 4: We have

$$
\int_{L} \Psi_{1} \cdot \boldsymbol{\Omega}_{p} \mathbf{w} d s \neq 0
$$

for $k=1$ and.

$$
\operatorname{det}\left[\begin{array}{cc}
\int_{L} \Psi_{1} \cdot \boldsymbol{\Omega}_{p} \mathbf{w}_{1} d s & \int_{L} \Psi_{1} \cdot \boldsymbol{\Omega}_{p} \mathbf{w}_{2} d s \\
\int_{L} \Psi_{2} \cdot \boldsymbol{\Omega}_{p} \mathbf{w}_{1} d s & \int_{L} \Psi_{2} \cdot \boldsymbol{\Omega}_{p} \mathbf{w}_{2} d s
\end{array}\right] \neq 0
$$

in the case $k=2$.

The opposite assumption is easily led to a contradiction by the pattern of Corollary 1 of $\S 11$

Now, let us consider the lineare space $\mathfrak{Q}$. of solutions of the problem $C^{*}$ with contact data belonging to the linear manifold

$$
\mathfrak{B}=\left\{\mathbf{w}_{0} / \mathbf{w}_{0}=A_{1} \mathbf{w}_{1}+A_{2} \mathbf{w}_{2}+\mathbf{h}, \quad \mathbf{h} \in \operatorname{ker} \boldsymbol{\Omega}_{p}\right\} .
$$

Lemma 4: The linear space $\mathfrak{Q}$ has the dimension $x_{h^{*}}+3$. A basis of $\mathfrak{Q}$ is given by the vectors

$$
\mathbf{V}\left(\mathbf{x} ; \boldsymbol{\Phi}_{1}\right), \ldots, \mathbf{V}\left(\mathbf{x} ; \boldsymbol{\Phi}_{x_{h^{*}+1}}\right), \quad \mathbf{V}_{1}, \mathbf{V}_{2},
$$

where the vectors $\boldsymbol{\Phi}_{j}\left(j=1, \ldots, x_{h}+1\right)$ are those of Corollary 3, but $\mathbf{V}_{1}, \mathbf{V}_{2}$ are given by (11.17).

Indeed, the vectors (12.24) are linearly independent and belong to $\mathfrak{Q}$. Otherwise, in consequence of $\operatorname{dim} \mathfrak{P}=x_{h^{*}}+2$ we have $\operatorname{dim} \mathfrak{Q} \leqq x_{h^{*}}+3$. The lemma is proved

Now; we consider the equation

$$
\boldsymbol{\Omega}_{p} \mathcal{A} \Phi=\boldsymbol{\Omega}_{p} \mathbf{w}+A_{1} \boldsymbol{\Omega}_{p} \mathrm{w}_{1}+A_{2} \boldsymbol{\Omega}_{p} \mathrm{w}_{2} .
$$

Because of Corollary $\dot{4}$, the constants $A_{i}$ can be chosen in such a way that the equation (12.25) has a solution $\overline{\boldsymbol{\Phi}}$. Using Corollary 3 , the solution $\tilde{\boldsymbol{\Phi}}$ can be assumed to satisfy the condition.

$$
\int_{L} \mathbf{c}_{\omega}{ }^{1} \cdot \dot{\varphi}^{0} d s=0
$$

Suppose that the physical condition (3.7) is fulfilled. Then we have

$$
\int_{L} \mathbf{c}_{\omega} \cdot \bar{\varphi}^{0} d s=0, \quad \text { in virtue of }(12.24) .
$$

Now, using Lemma 4, the existence of a *-regular solution of the problem $C^{*}$ is easily proved, provided that the physical condition (3.7) is satisfied. Thus, the following theorem is proved.

Theorem 2: Let $L \in C^{2, \beta}(0<\beta \leqq 1)$, and let the situation of the first example of $\S 3$ [2: Figure 1] occur. Furthermore, let the contact data satisfy the assumptions of $\S 2$, the compatibility conditions (1.6a) $-(1.6 \mathrm{x})$ and, additionally, the physical condition (3.7). 
Then there exists $a^{*}$-regular solution $\mathbf{u}$ of the problem $C^{*} . \mathbf{u}$ is representable in the form (11.14). $\Phi$ and the constants $A_{1}, A_{2}$ are determined by the integral equation (5.10) with the additional condition

$$
\int_{L} \mathbf{c}_{\omega}^{1} \cdot \varphi^{0} d s=0
$$

We still remark that the necessary and sufficient condition for solvability of $C^{*}$ in the considered case is not a consequence of solutions of the adjoint integral equation' system. Instead of this, by (12.14) the condition (3.7) causes the relation.

$$
\int_{L}^{1} \mathbf{c}_{\omega} \cdot \boldsymbol{\varphi}^{0} d s=\dot{0}
$$

which is responsible (together with $(12.26)$ ) for the *-regularity of the potential $\mathbf{V}(\mathbf{x} ; \boldsymbol{\Phi})$.

Finally, let us give the results for the remaining examples of $\S 3$.

In the first example of dimension 2 the solution $\Phi$ of

$$
\boldsymbol{\Omega}_{p} \mathscr{A} \boldsymbol{\Phi}=\boldsymbol{\Omega}_{p} \mathbf{w}+A_{1} \boldsymbol{\Omega}_{p} \mathbf{w}_{1}+A_{2} \boldsymbol{\Omega}_{p} \mathbf{w}_{2}
$$

satisfies the condition $\int \varphi^{0} d s=0$ if and only if the conditions (3.9) are fulfilled. Further, we have $\operatorname{dim} \mathfrak{M}=\ddot{\varkappa}_{h^{*}}+\stackrel{L}{2}=\operatorname{dim} \mathfrak{M}_{0^{*}}$. The above defined linear space has the dimension $x_{h^{*}}+4$. In analogy to Lemma 3 , the vectors $\mathbf{V}\left(\mathbf{x} ; \Phi_{1}\right), \ldots, \mathbf{V}\left(\mathbf{x} ; \boldsymbol{\Phi}_{x_{h^{\bullet}}+2}\right), \mathbf{V}_{1}, \nabla_{2}\left(\Phi_{1}, \ldots, \Phi_{x_{h^{\bullet}+2}}\right.$ form a basis_of $\mathfrak{I Z}$ ) generate the linear space $\mathfrak{Q}$. Without any restrictions there exists a solution of the equation (*). One obtains $a^{*}$-regular solution $\mathbf{u}$ of $C^{*}$ if and only if the conditions (3.9) are fulfilled.

In the second example of dimension 2 (see [2: Figure 3]), every solution of $(*)$ satisfies the relation $\int \mathrm{c}_{\omega}{ }^{\perp} \varphi^{0} d s=0$, if and only if $(3.10)$ holds. The adjoint homogeneous equation $\left(\Omega_{p} \mathscr{A}\right)^{\prime} \Psi=0$ hías the solution

$$
\begin{array}{r}
{\left[\begin{array}{c}
\left(\mathbf{s} \cdot \mathbf{c y}_{\mathbf{y}}{ }^{3}\right) \\
0 \\
0 \\
0
\end{array}\right], \quad y \in L_{2}} \\
\Psi_{1}(\mathbf{y})=\left\{\begin{array}{c}
{\left[\begin{array}{c}
0 \\
0 \\
\mathbf{c}_{\mathbf{y}}{ }^{3}
\end{array}\right], \quad \mathbf{y} \in L_{7}, \ldots} \\
{\left[\begin{array}{c}
0 \\
0 \\
0 \\
\left(\mathbf{n} \cdot \mathbf{c}_{\mathbf{y}}{ }^{3}\right)
\end{array}\right], \quad y \in L_{8}}
\end{array}\right.
\end{array}
$$

which gives rise to the condition (3.11). One can prove the sufficiency of (3.10) and (3.11) for the problem $C^{*}$ also in the considered case.

In the two cases of dimension 3 (see $\S 3[2$ : Figure, 4$]$ ), the conditions $(3.12)$ imply $\int_{L} \varphi^{0} d s=0$ for every solution of $(*)$. The adjoint homogeneous equation allows the following solutions:

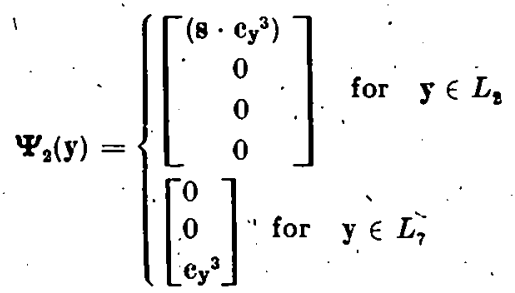


in the first case, and

$$
\Psi_{s}(y)=\left\{\begin{array}{l}
{\left[\begin{array}{c}
\left(8 \cdot c_{\omega}\right) \\
0 \\
0 \\
0
\end{array}\right], \text { for } y \in L_{2}} \\
{\left[\begin{array}{c}
0 \\
0 \\
c_{\omega}
\end{array}\right] \text { for } y \in L_{7}}
\end{array}\right.
$$

in the other one.

Finally, every solution $\Phi$ of $(*)$ in the last example of dimension 4 ( $\$ 3$ [2: Figure 5]) satisfies $\int \varphi^{0} d s=0$, if and only if the conditions (3.15) are valid. The vectors

$\boldsymbol{L}$

$$
\Psi_{4}(\mathbf{y})=\left\{\begin{array}{l}
{\left[\begin{array}{c}
\left(\mathbf{h} \cdot \mathbf{c}_{v}{ }^{3}\right) \\
0 \\
0 \\
0
\end{array}\right] \text { for } \mathbf{y} \in L_{5}} \\
{\left[\begin{array}{c}
0 \\
0 \\
c_{v}{ }^{3}
\end{array}\right] \text { for } y \in L_{7}}
\end{array}\right.
$$

and

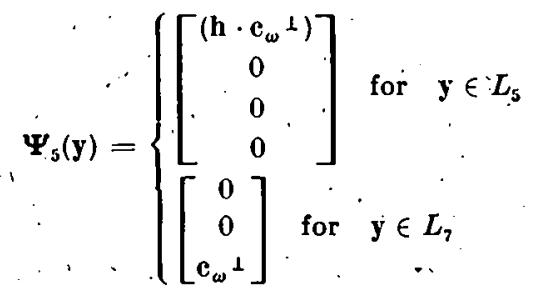

solve the adjoint homogeneous system.

The common result is the necessity and sufficiency of the physical conditions for solving problem $C^{*}$. Using the ideas sketched above, this result can also be established for the other conceivable situations of problem $C^{*}$.

\section{$\S 13 \varepsilon-$ and $\varepsilon^{*}$-regular solutions}

Studying the integral equation (5.10) in an arbitrary class $h\left(a_{j_{1}}, \ldots, a_{j_{\mathrm{o}}}\right)$, some results on the solution of the problems $C_{\varepsilon}$ and $C_{e}{ }^{*}$ may be expected in virtue of Theorem 4.2. Essentially, the general pattern of this theory resembles those considerations which have been developed in detail for boundary value problems of micropolar elasticity and thermoelasticity in [1] and [4].

Let

$$
m=\dot{\operatorname{dim}} \operatorname{ker}\left(\boldsymbol{\Omega}_{p} \mathcal{A}\right), \quad m^{\prime}=\operatorname{dim} \operatorname{ker}\left(\boldsymbol{\Omega}_{p} \mathcal{A}\right)^{\prime} .
$$

Then we have, because of Noether's theorems,

$$
m-m^{\prime}=x_{h} \text {. }
$$


Let the homogeneous problem have exactly $h$ linearly independent solutions. By the assumption on the integral equation (12.1) of $\S 12$, one easily obtains the relations

$$
\begin{aligned}
& \max \left(x_{h}+h, 0\right) \leqq m \leqq x_{h^{*}}+\dot{h}, \\
& \max \left(h,-\varkappa_{h}\right) \leqq m^{\prime} \leqq x_{h^{*}}-x_{h}+h .
\end{aligned}
$$

Thèse estimates can be improved. Indeed, for $h>0$ the solutions of the homogeneous problem $C^{*}\left(C_{\varepsilon}, C_{\varepsilon}^{*}\right)$ are regular. Therefore it is not difficult to' deduce

$$
\operatorname{dim} \operatorname{ker}\left(\boldsymbol{\Omega}_{\boldsymbol{p}} \mathscr{A}\right) \geqq h
$$

for every class $h$ and in any case. For this reason one gets

$$
\begin{aligned}
& \max \left(x_{h}+h, h\right) \leqq m \leqq \varkappa_{h^{*}}+h, \\
& \max \left(h, h-x_{h}\right) \leqq m^{\prime} \leqq x_{h^{*}}-x_{h}+h .
\end{aligned}
$$

Let

$$
q=x_{h^{*}}+h-m, \quad p=m^{\prime}-h .
$$

Then we have

$$
p+q=x_{h^{*}}-\left(m-m^{\prime}\right)=x_{h^{*}}-x_{h} .
$$

Now, one can show by the already mentioned argumentation of [1] that there exist exactly $h+p+q$ independent conditions for solvability of the considered problem $C_{e}^{*}-\left(C_{\varepsilon}\right)$. The following theorem holds.

Theorem 1: Let $L \in C^{2, \beta}(0<\beta \leqq 1)$. Let the homogeneous problem $C_{\varepsilon}{ }^{*}\left(C_{e}\right)$ have exactly $h$ linearly independent solutions. Let the contact data $\mathbf{f}, f, \mathbf{f}_{k}, f_{k}, l_{k}, \mathbf{g}, g, \mathrm{~g}_{k}, g_{k}, h_{k}$ satisfy the assumptions of $\S 2$, the compatibilityconditions $(1.6 \mathrm{a})-(1.6 \mathrm{x})$ and the physical solvability conditions of $\S \mathbf{3}$.

Then the problem $C_{\varepsilon}{ }^{*}\left(C_{\varepsilon}\right)$ permits an $\varepsilon^{*}$-regular ( $\varepsilon$-regular) solution $\mathbf{u}$ if and only if the contact data fulfil exactly $\varkappa_{h^{*}}-x_{h}$ additional solvability conditions.

The first $p$ of these additional solvability conditions are given by $p$ additional solutions of the homogeneous adjoint equation

$$
\left(\Omega_{p} \mathcal{A}\right)^{\prime} \Psi=0 .
$$

The remaining $q$ conditions can be interpreted as solvability conditions for the equation

$$
\mathcal{A} \boldsymbol{\Phi}=\mathbf{h}, \because \mathbf{h} \in \operatorname{ker} \boldsymbol{\Omega}_{p}
$$

in the class $h\left(a_{j_{1}}, \ldots, a_{j_{q}}\right)$.

\section{REFERENCES}

[1] MadL, J.: Existenzsätze und äquivalentë Integralgleichungen für allgemeine gemischte Randwertprobleme und Randliontaktprobleme der ebenen mikropolaren Elastizitätstheorie und Thermoelastizitätstheorie. In: I. Jentsch und J. Maul: Zur Elastizitäts- und Thermoelastizitätstheorie. Mathematical research vol. 4. Akademie-Verlag: Berlin 1970.

[2] - : Mixed contact problems in the plane elasticity I. Z. Anal. Anw. 2 (1983) 3, $207-234$.

[3] - : Zur Lösung von Aufgaben der ebenen Elastostatik in unendlichen Körpern. Beiträge zur Analysis 9 (1974), 55-60. 
[4] - : Beiträge zur Existenztheorie gemischter Randwert- und Kontaktaufnahmen der klassi-/ schen und mikropolaren Elastizitätștheoric und Thermoelastizitätstheorie. Diss. B: KarlMarx-Universität Leipzig 1979.

[5] - : Eine einheitliche Methode zur Lösung der ebenen Áufgaben der linearen Elastostatik. Schriftenr. ZIMM Akad, d. Wiss. d. DDR, Heft 24, Akademie-Verlag: Berlin 1976.

[6] Moskatelish vili, N. I.: Singular integral equations (Transl. from the Russian). P. Noordhoff Ltd: Groningen 1953.

[7] Веку , Н. П.: Системы сингуляных иитегральных уравненин. Москва 1970.

Manuskripteingang: 12.05 .1982

\section{VERFASSER:}

Doz. Dr. sc. Johannes MaUl

Sektion Mathematik der Karl-Marx-Universität Leipzig

DDR - 7010 Leipzig, Karl-Marx-Platz 